\title{
The Type la Supernova Rate in Redshift 0.5-0.9 Galaxy Clusters
}

\section{Citation}

Sharon, Keren, Avishay Gal-Yam, Dan Maoz, Alexei V. Filippenko, Ryan J. Foley, Jeffrey M. Silverman, Harald Ebeling, et al. 2010. "THE TYPE la SUPERNOVA RATE IN REDSHIFT 0.5-0.9 GALAXY CLUSTERS." The Astrophysical Journal 718 (2): 876-93. https:// doi.org/10.1088/0004-637x/718/2/876.

\section{Permanent link}

http://nrs.harvard.edu/urn-3:HUL.InstRepos:41399765

\section{Terms of Use}

This article was downloaded from Harvard University's DASH repository, and is made available under the terms and conditions applicable to Other Posted Material, as set forth at http:// nrs.harvard.edu/urn-3:HUL.InstRepos:dash.current.terms-of-use\#LAA

\section{Share Your Story}

The Harvard community has made this article openly available. Please share how this access benefits you. Submit a story.

Accessibility 


\title{
THE TYPE Ia SUPERNOVA RATE IN REDSHIFT 0.5-0.9 GALAXY CLUSTERS*
}

\author{
Keren Sharon $^{1,2}$, Avishay Gal-Yam ${ }^{3}$, Dan Maoz ${ }^{2}$, Alexei V. Filippenko ${ }^{4}$, Ryan J. Foley ${ }^{4,5,12}$, JefFrey M. Silverman ${ }^{4}$, \\ Harald Ebeling $^{6}$, Cheng-Jiun Ma ${ }^{6}$, Eran O. OfeK $^{7}$, Jean-Paul Kneib ${ }^{8}$, Megan Donahue ${ }^{9}$, Richard S. Ellis ${ }^{7}$, \\ Wendy L. Freedman ${ }^{10}$, Robert P. Kirshner ${ }^{5}$, John S. Mulchaey ${ }^{9}$, Vicki L. Sarajedini ${ }^{11}$, And G. Mark Voit ${ }^{9}$ \\ ${ }^{1}$ Kavli Institute for Cosmological Physics, University of Chicago, Chicago, IL 60637, USA \\ ${ }^{2}$ School of Physics and Astronomy, Tel Aviv University, Tel Aviv 69978, Israel \\ ${ }^{3}$ Benoziyo Center for Astrophysics, Faculty of Physics, Weizmann Institute of Science, Rehovot 76100, Israel \\ ${ }^{4}$ Department of Astronomy, University of California, Berkeley, CA 94720-3411, USA \\ ${ }^{5}$ Harvard-Smithsonian Center for Astrophysics, 60 Garden Street, Cambridge, MA 01238, USA \\ ${ }^{6}$ Institute for Astronomy, University of Hawaii, 2680 Woodlawn Drive, Honolulu, HI 96822, USA \\ ${ }^{7}$ Division of Physics, Mathematics, and Astronomy, California Institute of Technology, Pasadena, CA 91125, USA \\ ${ }^{8}$ Laboratoire d'Astrophysique de Marseille, CNRS-Université Aix-Marseille, 38 rue F. Joliot-Curie, 13388 Marseille Cedex 13, France \\ ${ }^{9}$ Department of Physics and Astronomy, BPS Building, Michigan State University, East Lansing, MI 48824, USA \\ ${ }_{10}$ Carnegie Observatories, 813 Santa Barbara Street, Pasadena, CA 91101, USA \\ ${ }^{11}$ Department of Astronomy, University of Florida, Gainesville, FL 32611, USA \\ Received 2009 October 26; accepted 2010 June 3; published 2010 July 7
}

\begin{abstract}
Supernova (SN) rates are potentially powerful diagnostics of metal enrichment and SN physics, particularly in galaxy clusters with their deep, metal-retaining potentials and relatively simple star formation histories. We have carried out a survey for SNe in galaxy clusters, at a redshift range of $0.5<z<0.9$, using the Advanced Camera for Surveys (ACS) on the Hubble Space Telescope. We reimaged a sample of 15 clusters that were previously imaged by ACS, thus obtaining two to three epochs per cluster in which we discovered five likely cluster SNe, six possible cluster Type Ia supernovae, two hostless SN candidates, and several background and foreground events. Keck spectra of the host galaxies were obtained to establish cluster membership. We conducted detailed efficiency simulations, and measured the stellar luminosities of the clusters using Subaru images. We derive a cluster $\mathrm{SN}$ rate of $0.35 \mathrm{SNu}_{B}{ }_{-0.12}^{+0.17}$ (statistical) \pm 0.13 (classification) \pm 0.01 (systematic) (where $\mathrm{SNu}_{B}=$ $\mathrm{SNe}\left(100 \mathrm{yr} 10^{10} L_{B, \odot}\right)^{-1}$ ) and $0.112 \mathrm{SNu}_{M}{ }_{-0.039}^{+0.055}$ (statistical) \pm 0.042 (classification) \pm 0.005 (systematic) (where $\left.\mathrm{SNu}_{M}=\mathrm{SNe}\left(100 \mathrm{yr} 10^{10} M_{\odot}\right)^{-1}\right)$. As in previous measurements of cluster $\mathrm{SN}$ rates, the uncertainties are dominated by small-number statistics. The $\mathrm{SN}$ rate in this redshift bin is consistent with the $\mathrm{SN}$ rate in clusters at lower redshifts (to within the uncertainties), and shows that there is, at most, only a slight increase of cluster SN rate with increasing redshift. The low and fairly constant SN Ia rate out to $z \approx 1$ implies that the bulk of the iron mass in clusters was already in place by $z \approx 1$. The recently observed doubling of iron abundances in the intracluster medium between $z=1$ and 0 , if real, is likely to be the result of redistribution of existing iron, rather than new production of iron.
\end{abstract}

Key words: galaxies: clusters: general - supernovae: general

Online-only material: color figures

\section{INTRODUCTION}

Quantifying the rates and properties of supernovae ( $\mathrm{SNe})$ in high-redshift galaxy clusters is important for several applications. In structure-formation studies, $\mathrm{SNe}$ play a crucial role in baryonic physics. Their energy deposition into the environment is relevant to both galaxy formation and star formation. Numerical simulations of galaxy formation now include feedback from SN explosions (e.g., Borgani et al. 2004; Kay et al.

\footnotetext{
* Based on observations made with the NASA/ESA Hubble Space Telescope, obtained at the Space Telescope Science Institute, which is operated by the Association of Universities for Research in Astronomy, Inc., under NASA contract NAS 5-26555. These observations are associated with programs GO-10493 and GO-10793, and with archival programs GO-9033, GO-9090, GO-9290, GO-9292, GO-9722, GO-9744, GO-9836, and GO-10509. Based in part on data collected at the Subaru telescope, which is operated by the National Astronomical Observatory of Japan. Some of the data presented herein were obtained at the W. M. Keck Observatory, which is operated as a scientific partnership among the California Institute of Technology, the University of California, and NASA; it was made possible by the generous financial support of the W. M. Keck Foundation.

${ }^{12}$ Clay Fellow.
}

2007; Nagai et al. 2007; Scannapieco et al. 2008; see Borgani et al. 2008a, 2008b for reviews), but the efficiency of this feedback is unknown.

In terms of cosmic metal-enrichment history, $\mathrm{SNe}$ are the sources of iron and other heavy elements that can be observed in the intracluster medium (ICM) and are detectable through X-ray observations (e.g., Balestra et al. 2007; Maughan et al. 2008; de Plaa et al. 2007). The abundances of these elements in the ICM depend on the integrated history of SN explosions (e.g., Maoz \& Gal-Yam 2004), as all of the elements produced during all stages of cluster formation and evolution must remain in the cluster due to its deep potential well. The abundances also depend on the efficiency with which matter is ejected from galaxies into the ICM, whether by SN-driven galactic winds (De Young 1978; White 1991; Renzini 1997; Borgani et al. 2008b; Sivanandam et al. 2009), by gas stripping due to ram pressure (Gunn \& Gott 1972; Mori \& Burkert 2000), or by galaxy-galaxy interactions (e.g., Clemens et al. 2000). SNe from a diffuse intergalactic stellar population may also be non-negligible contributors to the ICM enrichment (Gal-Yam \& Maoz 2000a, 2000b; Gal-Yam et al. 2003; Lin \& Mohr 2004; Tornatore et al. 2007). Measuring the properties and rates of $\mathrm{SNe}$ of all types in 
clusters as a function of redshift can thus shed light on galaxy and cluster formation.

Finally, cluster SN rates can provide clues for our understanding of Type Ia supernova (SN Ia) physics. It is widely agreed that $\mathrm{SNe}$ Ia are the thermonuclear explosions of nearChandrasekhar-mass carbon-oxygen white dwarfs in binary systems. However, the nature of the progenitor systems is still unknown, and several different channels have been proposed (see, e.g., Mannucci et al. 2008 for a recent overview). One prediction of a progenitor scenario that can be tested by observations is the delay-time distribution (DTD) between the formation of a stellar population and the SN Ia explosion of some of its members. In recent years, constraining the DTD has been attempted by comparing cosmic star formation history (SFH) to redshift-dependent rates of SNe Ia in the field (e.g., Gal-Yam \& Maoz 2004; Dahlen et al. 2004, 2008; Cappellaro et al. 2005; Neill et al. 2006; Botticella et al. 2008; Poznanski et al. 2007; Kuznetsova et al. 2008). A major complication in such measurements is the observational uncertainty in the SFH (e.g., Förster et al. 2006). In a further recent development, several studies have found evidence for the coexistence of two SN Ia explosion channels, a "prompt" channel that leads to an explosion within $\sim 10^{8} \mathrm{yr}$ of the formation of a progenitor binary system and a "delayed" one that occurs at least several Gyr after star formation and dominates in old stellar environments (Mannucci et al. 2005, 2006; Scannapieco \& Bildsten 2005; Sullivan et al. 2006b; Totani et al. 2008; Pritchet et al. 2008; Aubourg et al. 2008; Raskin et al. 2009; Maoz et al. 2010a; Brandt et al. 2010; Maoz \& Badenes 2010).

Galaxy clusters form unique environments for DTD studies. The stellar population in galaxy clusters is dominated by old stars in early-type galaxies, particularly in the core of the cluster (e.g., Visvanathan \& Sandage 1977; Renzini 2006) with a very small amount of star formation taking place, mainly in starforming galaxies at the outskirts of the clusters (e.g., Hansen et al. 2009; Porter et al. 2008; Bai et al. 2007; Saintonge et al. 2008; Loh et al. 2008). The fraction of star-forming galaxies increases with redshift (Butcher \& Oemler 1978, 1984), an effect that is independent of cluster richness (Hansen et al. 2009). Since clusters have little ongoing star formation, measuring the redshift-dependent SN Ia rate in clusters can isolate the delayed channel. Moreover, since the SFH in clusters is relatively simple compared to field galaxies, the DTD that can be deduced from the SN rate depends less strongly on the details of the assumed SFH (Maoz \& Gal-Yam 2004).

To date, the SN Ia rate as a function of redshift in clusters has not been well measured. Until recently, the few existing published rates relied on small numbers of detected $\mathrm{SNe}$, and the large uncertainties were dominated by small-number statistics. An intermediate/high- $z$ SN Ia rate was derived by Gal-Yam et al. (2002) using archival Hubble Space Telescope (HST) imaging of nine clusters, in which they discovered two or three likely cluster SNe. Their measured rates were $0.39_{-0.25}^{+0.59}$ and $0.80_{-0.40}^{+0.92}$ $\mathrm{SNu}_{B}$ at $z=0.25$ and $z=0.9$, respectively, where $\mathrm{SNu}_{B}$ denotes $\mathrm{SNe}\left(100 \mathrm{yr} 10^{10} L_{B, \odot}\right)^{-1}$. These rates correspond to roughly $0.11_{-0.07}^{+0.16}$ and $0.22_{-0.11}^{+0.25} \mathrm{SNu}_{M}$, respectively, where $\mathrm{SNu}_{M}$ denotes $\mathrm{SNe}\left(100 \mathrm{yr} 10^{10} M_{\odot}\right)^{-1}$ (see Section 8 ). Based on three cluster $\mathrm{SNe}$ and three possible cluster $\mathrm{SNe}$, Graham et al. (2008) derived an SN Ia cluster rate at $0.2<z<1.0$ from the CFHT Supernova Legacy Survey (SNLS) of $0.1_{-0.04}^{+0.09} \mathrm{SNu}_{M}$. Mannucci et al. (2008) have reanalyzed the Cappellaro et al. (1999) nearby SN sample to derive a local $(z<0.04)$ SN Ia cluster rate, based on $11 \mathrm{SNe}$, of $0.066_{-0.020}^{+0.027} \mathrm{SNu}_{M}$, which they found to be significantly higher than the corresponding rate in field elliptical galaxies, $0.019_{-0.008}^{+0.013} \mathrm{SNu}_{M}$. Sharon et al. (2007) reported a rate of $0.098_{-0.039}^{+0.059} \pm 0.009 \mathrm{SNu}_{M}$ at a slightly higher redshift, $0.06<z<0.19$, based on the Wise Observatory Optical Transient Survey (WOOTS) detection of six cluster SNe Ia (Gal-Yam et al. 2008). The Sloan Digital Sky Survey-II (SDSS-II) Supernova Survey has discovered thousands of SN candidates, and by cross-correlation of the confirmed SNe with SDSS cluster catalogs (Koester et al. 2007; Miller et al. 2005) measured cluster rates of $0.060_{-0.020-0.001}^{+0.027+0.002} \mathrm{SNu}_{M}$ at $z=0.084$ and $0.088_{-0.018-0.002}^{+0.022} \mathrm{SNu}_{M}$ at $z=0.225$ (Dilday et al. 2010).

Measurements of SN Ia cluster rates from larger surveys are ongoing. To name a few, the Palomar Transient Factory (Law et al. 2009; Rau et al. 2009) is expected to discover thousands of $\mathrm{SNe}$ in a footprint of $>8000 \mathrm{deg}^{2}$, which will also be used to measure cluster SN rates at low redshift. At very high redshifts, the Supernova Cosmology Project (SCP; PI: Perlmutter) has targeted 25 clusters with a 219-orbit HST multi-epoch program (GO-10496) in which about eight cluster SNe have been discovered (Dawson et al. 2009; Melbourne et al. 2007), permitting derivation of the cluster SN rate at $z \gtrsim 1$ (Barbary et al. 2010; K. H. Barbary et al. 2010b, in preparation).

Here, we present results of an $H S T$-based SN survey in galaxy clusters at $0.5<z<0.9$. Throughout the paper, we assume a flat cosmology, with parameters $\Omega_{\Lambda}=0.7, \Omega_{m}=0.3$, and $H_{0}=70 \mathrm{~km} \mathrm{~s}^{-1} \mathrm{Mpc}^{-1}$. Magnitudes are reported in the Vegabased system unless stated otherwise.

\section{HST OBSERVATIONS AND REDUCTIONS}

We were allocated $30 \mathrm{HST}$ orbits to reimage 15 highredshift galaxy clusters during two observation cycles (programs GO-10493 in cycle 14 and GO-10793 in cycle 15, PI: A. Gal-Yam). The clusters were selected to be X-ray bright, in the redshift range $0.5<z<0.9$, and to have been imaged with the Advanced Camera for Surveys (ACS) in the past. We also required that archival data for each target were non-proprietary at the time of our imaging, to ensure prompt detection of SN candidates. To enable comparison to archival data, the new images were obtained using the same filters as the archival ones, either the F814W filter ( $\sim I$ band) or the F775W filter ( $\sim i$ band). New observations were obtained at the same position angles as the archival ones, or rotated by $90^{\circ}, 180^{\circ}$, or $270^{\circ}$, to allow maximal overlap between the images. The archival data consist of observations from several $H S T$ programs; see Table 1, which also lists the other observations that were used in this survey, and the area of overlap between epochs. Exposure times were typically one orbit ( $2000 \mathrm{~s})$ per observing epoch in programs GO-10493, GO-10793, GO-9292, GO-9744, and GO-9836, and at least two orbits ( 4500 s) in programs GO-9033, GO-9090, GO-9290, GO-9722, and GO-10509. The 5 $\sigma$ detection limiting magnitudes for a point source are typically $\sim 26.4 \mathrm{mag}$ in the $I$ band and $\sim 26.9 \mathrm{mag}$ in the $i$ band in our single-orbit observations, and $\sim 27.5 \mathrm{mag}$ in the two-orbit archival $V$-band (F555W) images that exist for some of the fields. The precise detection probabilities as a function of magnitude for variable point sources are measured through simulations, as detailed below in Section 7.1.1.

We were able to obtain high-quality data for all but one cluster in the original list during cycle 14 . The missing cluster, MACSJ0025-1222, was withdrawn from our survey since it had not yet been successfully observed (GO-9722) at the time of our survey. It was replaced in our cycle 15 target list with another 
Table 1

Cluster Fields

\begin{tabular}{|c|c|c|c|c|c|c|c|c|c|c|c|c|c|c|c|c|}
\hline \multirow[t]{2}{*}{ Cluster } & \multirow[t]{2}{*}{$z^{\mathrm{a}}$} & \multicolumn{2}{|c|}{ Coordinates (J2000) } & \multicolumn{2}{|l|}{ Epoch $\mathrm{I}^{\mathrm{b}}$} & \multicolumn{5}{|c|}{ Epoch II (GO-10493) } & \multicolumn{5}{|c|}{ Epoch III (GO-10793) } & \multirow[t]{2}{*}{ Filter } \\
\hline & & R.A. & Decl. & Date (yyyy/mm) & GO & Date (yyyy/mm) & Area $^{c}$ & $\Delta t^{\mathrm{d}}$ & $L_{B}^{\mathrm{e}}$ & $M^{\mathrm{f}}$ & Date (yyyy/mm) & Area $^{c}$ & $\Delta t^{\mathrm{g}}$ & $L_{B}^{\mathrm{e}}$ & $M^{\mathrm{f}}$ & \\
\hline MACSJ2214-1359 & 0.503 & 221457.34 & -140012.2 & $2003 / 10$ & 9722 & $2005 / 08$ & 8.34 & 100.5 & 4.4 & 14.2 & & 0 & 0 & 0 & 0 & F814W \\
\hline MACSJ0911+1746 & 0.505 & 091111.18 & +174634.8 & $2004 / 03$ & 9722 & $2005 / 10$ & 8.44 & 93.4 & 2.2 & 6.9 & $2006 / 12$ & 10.3 & 101.2 & 2.5 & 7.6 & F814W \\
\hline MACSJ0257-2325 & 0.505 & 025708.83 & -232603.3 & 2004/01 & 9722 & $2005 / 08$ & 8.05 & 75.4 & 2.1 & 6.4 & $2006 / 08$ & 10.5 & 99.0 & 2.4 & 7.5 & F814W \\
\hline MS0451.6-0305 & 0.538 & 045410.48 & -030138.5 & $2004 / 01$ & 9836 & $2005 / 07$ & 7.96 & 83.3 & 3.0 & 9.4 & $\ldots$ & 0 & 0 & 0 & 0 & F814W \\
\hline MACSJ1423+2404 & 0.543 & 142348.60 & +240449.1 & 2004/01 & 9722 & $2006 / 03$ & 9.13 & 94.4 & 3.1 & 9.6 & & 0 & 0 & 0 & 0 & F814W \\
\hline MACSJ1149+2223 & 0.544 & 114935.51 & +222404.2 & 2004/04 & 9722 & $2006 / 05$ & 9.67 & 85.0 & 5.2 & 15.4 & & 0 & 0 & 0 & 0 & F814W \\
\hline MACSJ0717+3745 & 0.546 & 071732.93 & +374505.4 & 2004/04 & 9722 & $2005 / 10$ & 8.67 & 83.4 & 5.7 & 16.9 & $2006 / 10$ & 10.5 & 72.2 & 6.2 & 18.5 & F814W \\
\hline MS0016.5+1654 ${ }^{\mathrm{h}}$ & 0.546 & 001832.80 & +162606.9 & $2002 / 01$ & 9292 & $2006 / 06$ & 6.65 & 56.4 & 1.3 & 3.7 & $\ldots$ & 0 & 0 & 0 & 0 & F775W \\
\hline MACSJ0025-1222 & 0.584 & 002530.23 & -122243.0 & $2004 / 10$ & 9722 & $\ldots$ & 0 & 0 & 0 & 0 & $\ldots$ & 0 & 0 & 0 & 0 & F814W \\
\hline MACSJ2129-0741 & 0.589 & 212926.30 & -074126.2 & 2003/09 & 9722 & $2005 / 06$ & 8.64 & 70.1 & 1.7 & 5.1 & $\ldots$ & 0 & 0 & 0 & 0 & F814W \\
\hline MACSJ0647+7015 & 0.591 & 064749.78 & +701456.4 & $2004 / 12$ & 9722 & $2006 / 02$ & 8.41 & 71.6 & 3.9 & 10.3 & 2006/11 & 8.54 & 74.6 & 3.9 & 10.4 & F814W \\
\hline SDSSJ1004+4112 & 0.680 & 100434.72 & +411245.0 & $2004 / 04$ & 9744 & $2005 / 12^{\mathrm{d}}$ & 4.65 & 59.0 & 2.3 & 12.6 & $2007 / 01$ & 9.68 & 58.6 & 2.3 & 12.6 & F814W \\
\hline MACSJ0744+3927 & 0.697 & 074452.58 & +392726.7 & $2004 / 02$ & 9722 & $2005 / 12$ & 8.43 & 58.2 & 4.1 & 11.1 & $2006 / 12$ & 8.97 & 55.6 & 4.0 & 10.9 & F814W \\
\hline MS1054.4-0321 & 0.833 & 105700.20 & -033727.0 & $2002 / 12$ & 9290 & 2006/01 & 9.11 & 43.3 & 3.8 & 11.3 & 2007/01 & 10.4 & 43.5 & 3.9 & 11.6 & F775W \\
\hline CL0152-1357 & 0.835 & 015243.00 & -135720.0 & $2002 / 11$ & 9290 & 2005/06 & 10.0 & 32.4 & 2.7 & 8.0 & 2006/09 & 7.20 & 34.8 & 2.5 & 7.5 & F775W \\
\hline CLJ1226.9+3332 & 0.888 & 122658.21 & +333249.4 & 2003/04 & 9033 & 2006/01 & 10.7 & 35.8 & 1.1 & 3.6 & 2007/01 & 10.5 & 37.8 & 1.7 & 5.5 & F814W \\
\hline
\end{tabular}

Notes.

${ }^{a}$ References for cluster redshifts are Ebeling et al. (2007) for the MACS clusters, Ebeling et al. (2001) for CL0152-1357 and CLJ1226.9+3332, Tran et al. (1999) for MS1054.4-0321, and Oguri et al. (2004) for SDSS1004+41.

${ }^{\mathrm{b}} \Delta t, L_{B}$, and $M$ of epoch I are the same as those of epoch II.

${ }^{c}$ Overlapping area between epochs $\left(\operatorname{arcmin}^{2}\right)$. The imaging area of ACS is $10.5 \operatorname{arcmin}^{2}$.

d GO-10509.

e Stellar luminosity within the search area $\left[10^{12} L_{B, \odot}\right]$ (see Section 7.2).

${ }^{\mathrm{f}}$ Stellar mass within the search area $\left[10^{12} M_{\odot}\right]$ (see Section 7.4).

g Visibility time (days).

${ }^{\mathrm{h}}$ MS0016.5+1654 and MS0451.6-0305 are also MACS clusters, MACSJ0018.5+1626 and MACSJ0454.1-0300, respectively. 
cluster, SDSSJ1004+4112, that had already been observed twice by HST (cycle 12, GO-9744; cycle 14, GO-10509) and fits our selection criteria. During cycle 15 , the halt in operations of ACS caused the early termination of our program; six of the targets were not imaged. We therefore have three epochs for nine clusters, and only two epochs for six clusters.

Each new epoch was split into four dithered subexposures. The subexposures of each epoch were reduced using the standard HST/ACS pipeline, and combined using the Multidrizzle routine (Koekemoer et al. 2002) to remove cosmic-ray hits, dead or hot pixels, and other artifacts such as trails from satellites that crossed the field of view, with a square kernel, pixfrac $=1.0$ and scale=INDEF. Images from consecutive epochs of the same field were then aligned and subtracted from each other to form a difference image. Specifically, transient candidates were searched for in epoch I compared to epoch II, in epoch II compared to epoch I, and in epoch III compared to epoch II. We note that point-spread function (PSF) matching was not required (see Section 7.1.1 for more details).

Each difference image was searched by eye promptly after the observation, and all transient or variable candidates were noted. Obvious variable stars and known active galactic nuclei (AGNs) were removed from the follow-up list at this stage. Table 2 lists the remaining candidates. Assuming that SN production follows light (e.g., Förster \& Schawinski 2008), a galaxy was considered to be a candidate's host if the candidate was within its $2 \sigma$ isophotal contour, defined as the contour along which the galaxy flux per pixel is $2 \sigma$ above the background fluctuations, which in practice means the candidate is seen clearly embedded in the galaxy light. From the areas enclosed by these isophotal contours, the probability for a chance association in a given image is $<2 \%$. Three of the thirty-seven candidates do not satisfy this criterion, yet they were also chosen as likely candidate-host associations due to their small projected distances to their putative hosts, less than twice the radius of the $2 \sigma$ isophotal contour. In these cases, the probability for a chance association is $6 \%$. When more than one galaxy could be a likely host, follow-up spectroscopy was scheduled for the additional galaxies as well.

As detailed below and shown in Figure 1, among the 37 candidates, at least six are likely cluster events based on their host-galaxy redshifts (Figure 2), five of which are likely $\mathrm{SNe}$ Ia, and one is likely a core-collapse (CC) SN. Two candidates have ambiguous hosts and are possible cluster $\mathrm{SNe}$ Ia. Among the other candidates (Figure 3), eight are background transients (BG), eight are foreground events (FG), and three proved to be AGNs. Two candidates have no apparent host, and are possible cluster SNe Ia. The host galaxies of the remaining eight candidates are not confirmed by spectroscopy, and their classification is also discussed below. Of these eight unconfirmed candidates, four are possible cluster $\mathrm{SNe}$ Ia. The remaining four were variable (rather than transient) point sources that were detected at all epochs and are not clearly associated with any host galaxy, and are therefore probably quasars or Galactic variable stars.

\section{SUBARU IMAGES}

Our data analysis and derivation of SN rates made use of auxiliary ground-based data (see Section 7). Eleven of the clusters in our survey were imaged as part of a study of MACS clusters (Ebeling et al. 2007) using the SuprimeCam wide-field imager mounted on the Subaru $8.2 \mathrm{~m}$ telescope at Mauna Kea (Miyazaki et al. 2002), covering a field of view of $\sim 34^{\prime} \times 27^{\prime}$

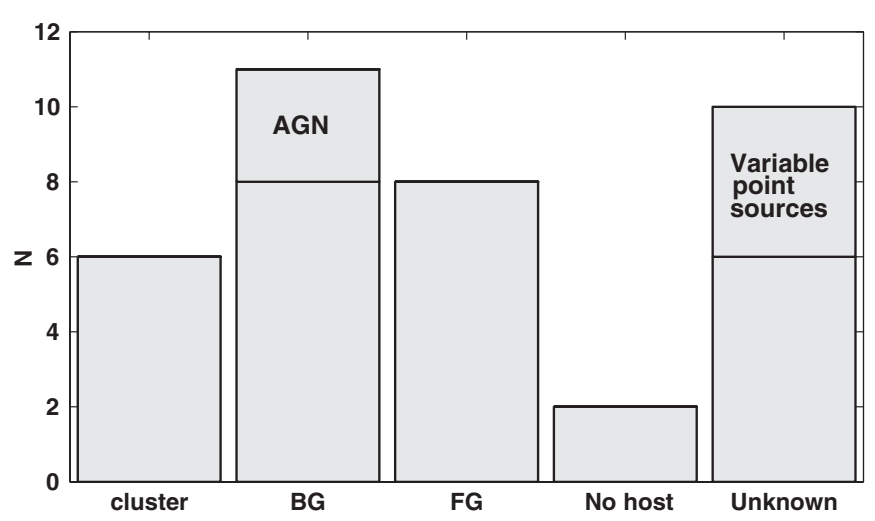

Figure 1. Candidate classification. "BG" and "FG" denote background and foreground events, respectively.

per cluster. Table 3 lists the clusters for which we have obtained Subaru data, the observation dates, and the exposure times. The $2.5 \sigma$ limiting magnitude is typically $R=25.7 \mathrm{mag}(\mathrm{AB})$. Details regarding the Subaru data, including data reduction and photometry, can be found in Kartaltepe et al. (2008). Briefly, the observations took place between 2000 and 2007 in variable conditions, with seeing ranging from $0{ }^{\prime} .6$ to $1^{\prime \prime} .1$. The data were reduced with the standard SuprimeCam pipeline (Donovan 2007). Photometric zero points were derived from overlap with the SDSS, or (for clusters outside the SDSS footprint) using 3 s exposures of nearby SDSS fields interlaced between the cluster observations. The zero-point uncertainty is $\sim 0.1 \mathrm{mag}$, and results from the different Subaru and SDSS filter passbands, and from the non-simultaneity of the interlaced calibration photometry.

Object catalogs for each cluster were created using SExtractor (ver. 2.4.3; Bertin \& Arnouts 1996) in "dual-image" mode (i.e., detecting objects in one image, while performing photometry on another) with the $R$-band image as the reference detection image. Star/galaxy separation was based on the SExtractor parameter MU_MAX (peak surface brightness above the background level). Since the light distribution of a source (e.g., its half-light radius) scales with magnitude, stars and other point sources populate a well-defined locus in an MU_MAX/MAG_AUTO plane, and can be excluded from the catalog (e.g., Bardeau et al. 2005). In addition, objects with peaks sharper than the PSF are not real astronomical objects, and can be flagged as artifacts. These catalogs were used as supplementary information for candidate classification (Section 5) and for measuring the cluster stellar luminosities (Section 7.2).

\section{HOST-GALAXY SPECTROSCOPY}

Nearly all of the SN candidates were too faint to be observed spectroscopically, even at the time of discovery, and even more so when the pre-allocated ground-based follow-up observing time arrived. Cluster membership was therefore established through spectroscopy of the host galaxy. In Table 2, we summarize the spectroscopic information acquired for each of the candidates. Follow-up spectra were obtained primarily using the $10 \mathrm{~m}$ Keck telescopes in Hawaii, either with the Low Resolution Imaging Spectrometer (LRIS; Oke et al. 1995) or the Deep Imaging Multi-Object Spectrograph (DEIMOS; Faber et al. 2003), in longslit or multislit configuration. The target lists were selected from the HST image and supplemented by other interesting objects in the field, such as gravitationally 

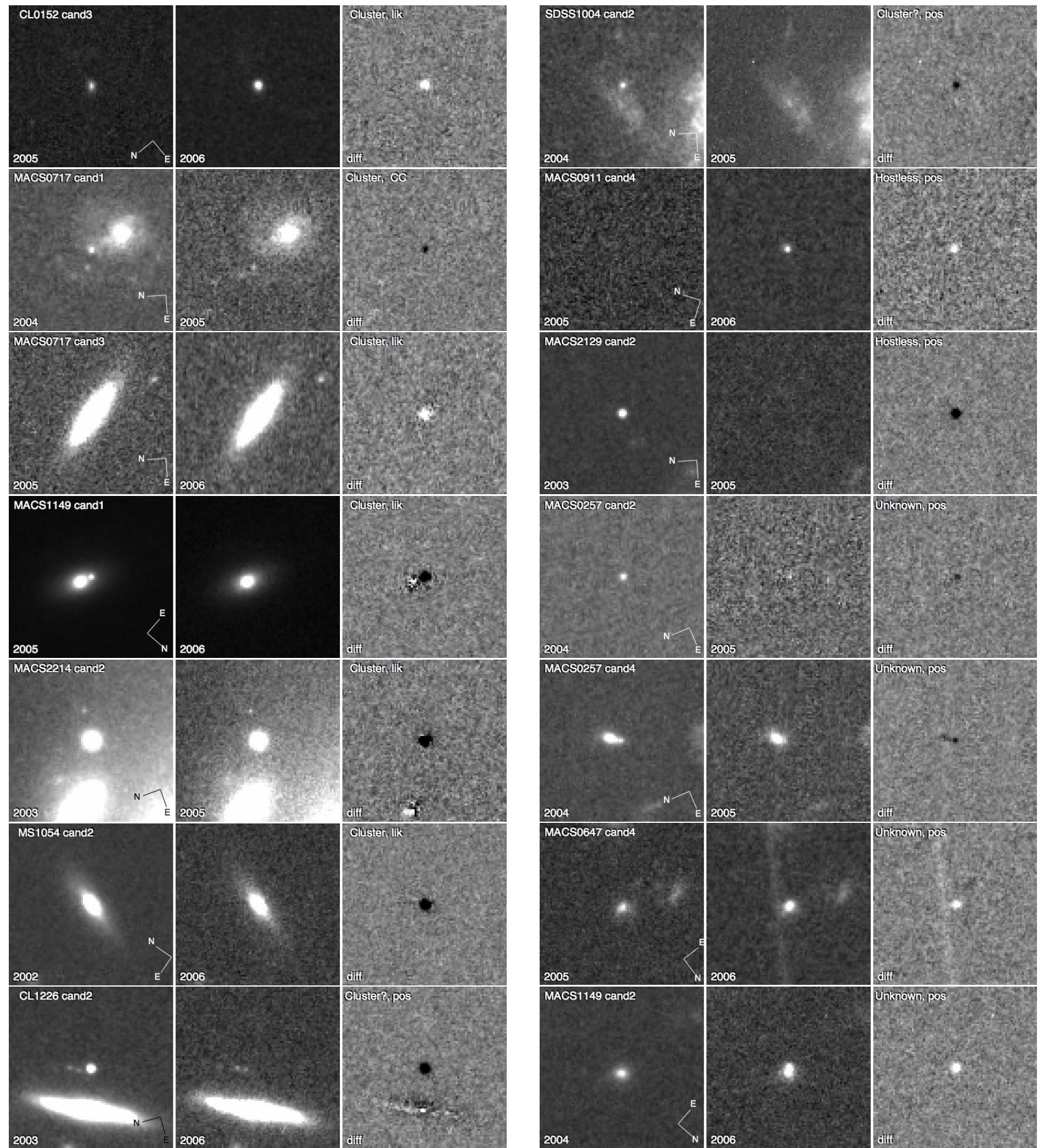

Figure 2. Discovery images of the possible and likely cluster-member SN candidates. In each row of three thumbnails, we show the first and second epochs in the left and middle frames, respectively. In the right panel, we show the difference, as a subtraction of the earlier epoch from the later epoch. The frames are each 4.4 wide, and centered on the transient candidate.

lensed galaxies and cluster members. For DEIMOS multislit spectroscopy, we filled the field of view with additional targets drawn from wide-field imaging with Subaru or SDSS. Table 2 lists the dates, instrument, and observers of each spectroscopic observation.

Multislit observations were reduced and analyzed as follows. After standard bias and flatfield corrections, we combined all the observations of the same field into a deep, cosmic-ray- cleaned two-dimensional spectrum. We compared each multislit spectrum with known night-sky lines, and calibrated the wavelength range using the IRAF ${ }^{13}$ tasks IDENTIFY, FITCOORDS, and TRANSFORM. Finally, we used the IRAF task APALL to

\footnotetext{
13 IRAF (Image Reduction and Analysis Facility) is distributed by the National Optical Astronomy Observatories, which are operated by AURA,
} Inc., under cooperative agreement with the National Science Foundation. 

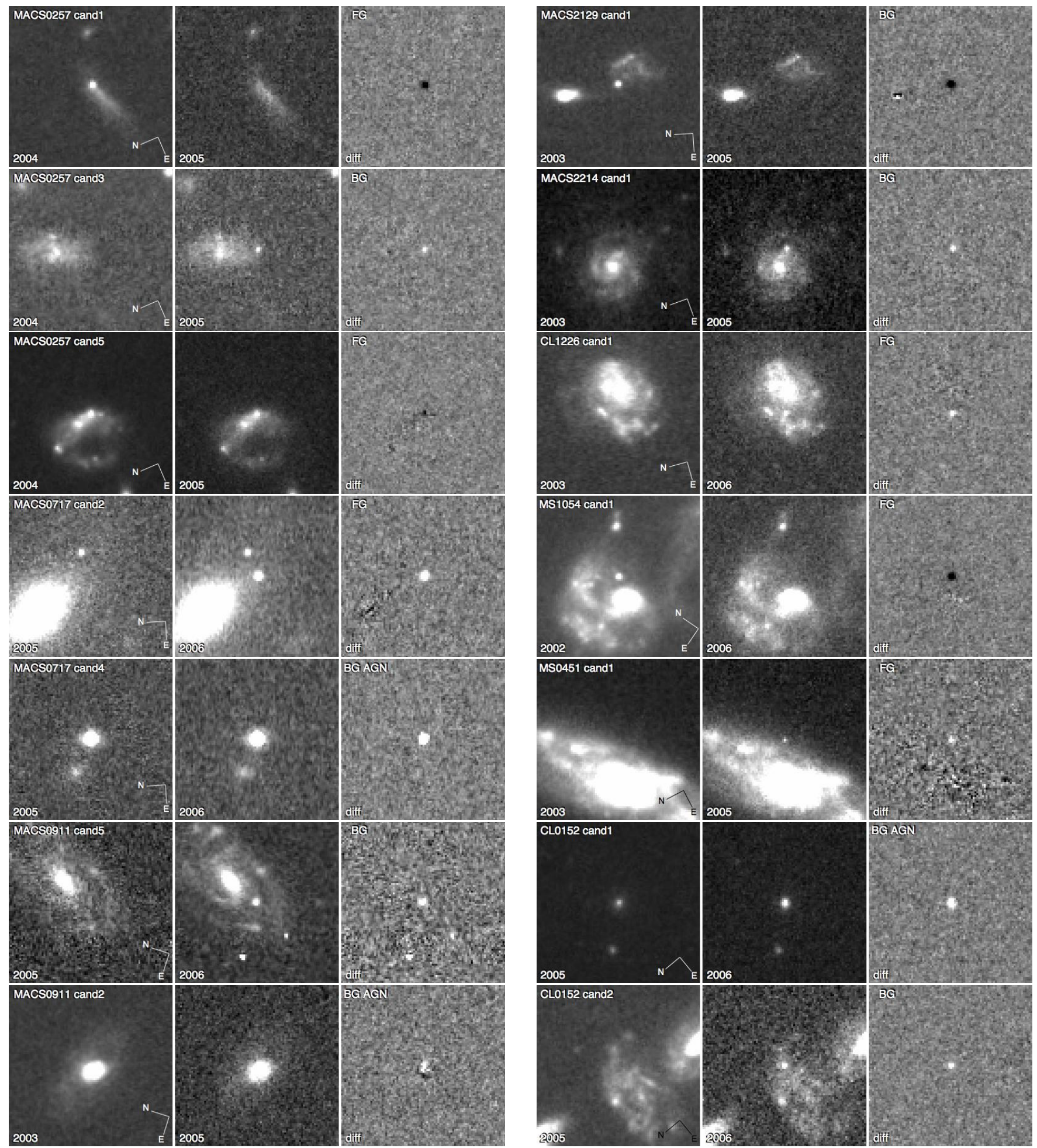

Figure 3. Same as Figure 2, but for non-cluster candidates.

subtract the background, trace the continuum of the object, and extract the one-dimensional spectrum from the calibrated image. ${ }^{14}$ The resulting spectrum was rebinned and searched for common galaxy emission and absorption lines. Longslit data were reduced in a similar manner, and in addition were also flux calibrated using spectra of standard stars from the same observing nights. This process allows, in principle, a comparison of the spectral shape of the object with those of template spectra, and derivation of a redshift even in the absence of emission or absorption features. In practice, the continuum signal from the host galaxies was generally weak, and the redshift determination is based on emission or absorption lines.

${ }^{14}$ Some of the spectra were reduced using tools developed in the MATLAB environment (Ofek et al. 2006). 


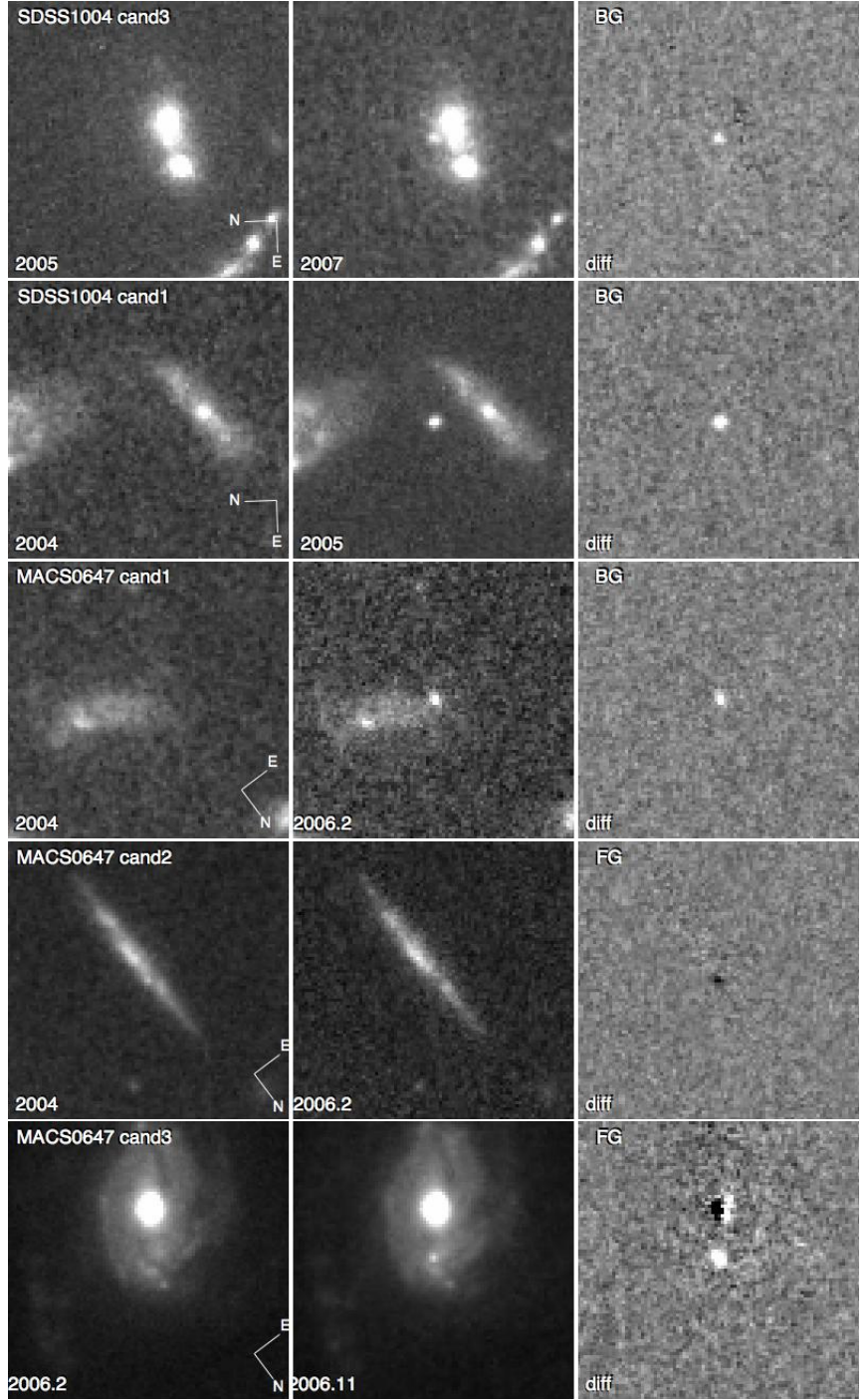

Figure 3. (Continued)

\section{CANDIDATE CLASSIFICATION}

As our transient candidates are not spectroscopically confirmed, we must consider the possibility that some or all of them are not SNe. The survey discussed in this paper is similar to the one reported by Gal-Yam et al. (2002), which was based on archival HST images of galaxy clusters, obtained with the Wide Field Planetary Camera 2 (WFPC2). While our survey is superior in resolution to the WFPC2 survey, and has a more uniform observation scheme, both surveys reach a similar depth, the search methods and efficiency simulations are done in the same manner, and the time span between epochs is similar. As discussed by Gal-Yam et al. (2002), transient events that could, in principle, mimic SNe include solar-system objects, variable stars in our Galaxy or in other galaxies, AGNs, or gamma-ray burst (GRB) afterglows. As Gal-Yam et al. (2002) argue, most of these transients cannot be confused with SNe. First, all but two of the candidates are clearly associated with galaxies. The probability for a chance association is small, $<2 \%-6 \%$ (see Section 2). The proper motions of asteroids or Kuiper-Belt objects would have been detected in our long-exposure images. With the exposure span within one HST orbit (typically $\sim 45$ minutes), we can detect proper motions greater than $\sim 0$. $015 \mathrm{hr}^{-1}$. At the dates and coordinates of our observations, the parallax of a Kuiper-Belt object at $50 \mathrm{AU}$ due to the Earth's motion is more than 0'.4, well above our detection limit. Variable stars in other galaxies are too faint to mimic SNe.

Variable stars in our own Galaxy, to be undetected in one of the epochs, would have to be distant. For example, an M5 flare star with absolute magnitude $M_{I}=9$ mag in its quiescent state (Allen 1973) would have to be at a distance $>40 \mathrm{kpc}$ to be fainter than our $I=27$ mag detection limit. The local density of halo stars with $0.09<M / M_{\odot}<0.71$ is of order $10^{-4} \mathrm{pc}^{-3}$ (Gould et al. 1998). A number of recent studies (see, e.g., Cignoni et al. 2007 and references therein) find an approximately $r^{-3}$ profile for the outer halo, and thus the stellar density at $40 \mathrm{kpc}$ is $10^{-4}(8.5 \mathrm{kpc} / 40 \mathrm{kpc})^{3} \approx 10^{-6} \mathrm{pc}^{-3}$. Integrating this density from $40 \mathrm{kpc}$ to, say, $80 \mathrm{kpc}$ gives a surface density of $\sim 10^{4} \mathrm{deg}^{-2}$. There could thus be several hundred outer-halo late-type stars in the 15 ACS fields. Based on this, the two hostless candidates we have found could, in principle, be distant Galactic M stars that had flared into visibility on one epoch. However, the flare would have had to be by $\gtrsim 2-2.5$ mag to bring the object from below the detection limit, $I=27 \mathrm{mag}$, to the observed brightnesses of the two hostless transients, $I=25.1$ and $I=24.4 \mathrm{mag}$, respectively. Although stellar flares can be bright in the ultraviolet, and reach amplitudes of $\Delta U \approx 5 \mathrm{mag}$, their amplitudes in red optical bands are much smaller (e.g., Eason et al. 1992; Allred et al. 2006; Zhilyaev et al. 2007), generally $\Delta I \lesssim 1 \mathrm{mag}$. Furthermore, a recent SDSS-based study by Kowalski et al. (2009) of M-dwarf flaring frequency and magnitude finds that the flaring duty cycle is strongly correlated with Galactic height. Beyond 300 pc above the disk, only $10^{-5}$ of their individual observations, which have cadences of several days, catch an $\mathrm{M}$ star in its flaring phase. Thus, apart from the implausibility of a large-amplitude $I$-band flare, there is only a $\sim 10^{-3}$ probability to start with that any of the $\sim 100$ outer-halo $\mathrm{M}$ stars in the ACS fields would be caught flaring in our observations. It is therefore highly unlikely that the hostless candidates are optical flares of Galactic stars.

For candidates that are in the centers of their associated hosts, an AGN nature would have been revealed by our spectroscopy. Since GRBs are often associated with some CC SNe (Galama et al. 1998; Hjorth et al. 2003; Stanek et al. 2003; Malesani et al. 2004; Pian et al. 2006; see Woosley \& Bloom 2006 for a review), we argue that in addition to being unlikely, they would have affected only our classification as SNe Ia or CC SNe, not the identification as SNe. We thus conclude that essentially all of our transient candidates are bona fide $\mathrm{SNe}$.

\section{CLASSIFICATION OF CLUSTER SN CANDIDATES}

Cluster membership of an SN candidate was decided according to the SN host-galaxy redshift and the cluster velocity dispersion (Ebeling et al. 2007). We classified SN candidate hosts as cluster members if their redshift indicated that their velocity is within $2 \sigma$ of the cluster recession velocity, where $\sigma$ is the velocity dispersion of the cluster. We note that the velocity dispersion of galaxies in clusters as massive as those in our sample is relatively high; the mean value for MACS clusters in our sample is $\sim 1300 \mathrm{~km} \mathrm{~s}^{-1}$ (Ebeling et al. 2007).

Next, although we have no spectroscopic classification, some clues about the type of the SN candidates exist. Since CC SNe are exceedingly rare in non-star-forming environments (e.g., Hakobyan et al. 2008), we classify all non-AGN candidates in cluster early-type galaxies as $\mathrm{SNe}$ Ia. The high resolution of ACS allows classification of resolved host galaxies through morphology, which can be supported by color information 
Table 2

SN Candidates

\begin{tabular}{|c|c|c|c|c|c|c|c|c|c|c|}
\hline \multicolumn{4}{|c|}{ Source } & \multirow{2}{*}{$\begin{array}{c}\text { Discovery } \\
\text { Epoch }\end{array}$} & \multicolumn{5}{|c|}{ Host } & \multirow[t]{2}{*}{ Classification } \\
\hline Name & R.A. $(\mathrm{h} \mathrm{m} \mathrm{s})$ & Decl. $\left({ }^{\circ} \prime \prime \prime\right)$ & $(\mathrm{mag})^{\mathrm{b}}$ & & R.A. $(\mathrm{h} \mathrm{m} \mathrm{s})$ & Decl. $\left({ }^{\prime \prime \prime \prime}\right)$ & (mag) & Morphology & $\operatorname{Spec} z(\operatorname{Ref})^{c}$ & \\
\hline MACSJ0257-2325-cand1 & 02:57:08.479 & $-23: 24: 24.38$ & 25.4 & 2004 Jan & 02:57:08.507 & $-23: 24: 24.55$ & 22.9 & Irr & $0.3294(\mathrm{O} 7)$ & Foreground \\
\hline MACSJ0257-2325-cand2 & $02: 57: 12.425$ & $-23: 27: 05.08$ & 26.8 & 2004 Jan & $02: 57: 12.425$ & $-23: 27: 05.08$ & $\gtrsim 27$ & Unresolved & $\mathrm{N} / \mathrm{A}$ & Possible cluster SN Ia \\
\hline MACSJ0257-2325-cand3 & 02:57:10.005 & $-23: 27: 14.52$ & 25.8 & 2005 Aug & 02:57:09.983 & $-23: 27: 13.54$ & 22.3 & Spiral & 0.73 (FF1) & Background \\
\hline MACSJ0257-2325-cand4 & 02:57:11.476 & $-23: 27: 19.72$ & 26.5 & $2004 \mathrm{Jan}$ & 02:57:11.464 & $-23: 27: 19.45$ & 23.6 & $\mathrm{E}$ & & Possible cluster SN Ia \\
\hline MACSJ0257-2325-cand5 & 02:57:06.904 & $-23: 27: 46.79$ & 26.3 & 2004 Jan & 02:57:06.947 & $-23: 27: 46.44$ & 20.9 & Spiral & $0.4039(\mathrm{O} 7)$ & Foreground \\
\hline MACSJ0647+7015-cand1 & $06: 47: 30.004$ & $+70: 14: 54.53$ & 25.8 & $2006 \mathrm{Feb}$ & $06: 47: 29.79$ & $+70: 14: 54.12$ & 22.5 & Spiral/Irr & $0.619(\mathrm{O} 8)$ & Background \\
\hline MACSJ0647+7015-cand2 & $06: 47: 38.030$ & $+70: 16: 16.72$ & 26.8 & $2004 \mathrm{Dec}$ & $06: 47: 38.024$ & $+70: 16: 16.05$ & 22.2 & Spiral & $0.495(\mathrm{O} 8)$ & Foreground \\
\hline MACSJ0647+7015-cand3 & $06: 47: 49.824$ & $+70: 15: 31.19$ & 24.6 & $2006 \mathrm{Nov}$ & $06: 47: 49.901$ & $+70: 15: 30.40$ & 18.6 & Spiral & $0.365(\mathrm{O} 8)$ & Foreground \\
\hline MACSJ0647+7015-cand4 & $06: 47: 59.661$ & $+70: 15: 19.51$ & 25.2 & 2006 Nov & $06: 47: 59.626$ & $+70: 15: 19.37$ & 23.7 & Unclear & & Possible cluster SN Ia \\
\hline MACSJ0717+3745-cand1 & 07:17:38.90 & $+37: 45: 20.8$ & 26.3 & 2004 Apr & $07: 17: 38.855$ & $+37: 45: 20.07$ & 21.7 & Spiral & $0.55(\mathrm{FF} 2)$ & Cluster, $\mathrm{CC}$ \\
\hline MACSJ0717+3745-cand2 & 07:17:31.444 & $+37: 44: 36.12$ & 24.4 & 2006 Oct & $07: 17: 31.519$ & $+37: 44: 37.58$ & 20.0 & E & 0.4915 (MS) & Foreground \\
\hline MACSJ0717+3745-cand3 & $07: 17: 41.444$ & $+37: 44: 10.54$ & 24.3 & 2006 Oct & $07: 17: 41.444$ & $+37: 44: 10.54$ & 20.6 & $\mathrm{E}$ & $0.538(\mathrm{MS})$ & Cluster SN Ia \\
\hline MACSJ0717+3745-cand4 & $07: 17: 40.548$ & $+37: 45: 06.60$ & 23.3 & 2006 Oct & $07: 17: 40.548$ & $+37: 45: 06.60$ & 21.6 & Compact & 2.084 (FSP) & Background AGN \\
\hline MACSJ0911+1746-cand2 & 09:11:16.407 & $+17: 47: 40.04$ & 24.9 & 2005 Oct & 09:11:16.407 & $+17: 47: 40.04$ & 21.6 & So & $0.88(\mathrm{E})$ & Background AGN \\
\hline MACSJ0911+1746-cand4 & 09:11:16.959 & $+17: 46: 48.42$ & 25.1 & $2006 \mathrm{Dec}$ & & & $\ldots$ & No host & $\ldots$ & Hostless, possible cluster SN Ia \\
\hline MACSJ0911+1746-cand5 & 09:11:19.290 & $+17: 46: 09.27$ & 25.2 & 2006 Dec & 09:11:19.269 & $+17: 46: 10.07$ & 21.0 & Spiral & 0.842 (FF4) & Background \\
\hline MACSJ1149+2223-cand1 & $11: 49: 33.20$ & $+22: 24: 29.90$ & 23.9 & 2004 Apr & $11: 49: 33.147$ & $+22: 24: 30.35$ & 20.0 & $\mathrm{E}$ & 0.553 (FF5) & Cluster SN Ia \\
\hline MACSJ1149+2223-cand2 & $11: 49: 35.734$ & $+22: 22: 18.34$ & 24.5 & 2006 May & $11: 49: 35.726$ & $+22: 22: 18.41$ & 23.6 & E & $\ldots$ & Possible cluster SN Ia \\
\hline MACSJ2129-0741-cand1 & $21: 29: 28.356$ & $-07: 41: 34.44$ & 25.0 & 2003 Sep & $21: 29: 28.321$ & $-7: 41: 34.77$ & 23.1 & Irr & $0.87(\mathrm{GY})$ & Background \\
\hline MACSJ2129-0741-cand2 & $21: 29: 24.993$ & $-07: 42: 22.76$ & 24.4 & 2003 Sep & & & $\ldots$ & No host & & Hostless, possible cluster SN Ia \\
\hline MACSJ2214-1359-cand1 & $22: 14: 59.353$ & $-13: 58: 15.28$ & 26.0 & 2005 Aug & $22: 14: 59.382$ & $-13: 58: 14.92$ & 20.9 & Spiral & $0.582(\mathrm{FF} 1)$ & Background \\
\hline MACSJ2214-1359-cand2 & $22: 14: 57.012$ & $-14: 00: 11.49$ & 23.7 & 2003 Oct & $22: 14: 57.012$ & $-14: 00: 11.49$ & 19.6 & $\mathrm{E}$ & 0.503 (GYS) & Cluster SN Ia \\
\hline CLJ1226.9+3332-cand 1 & $12: 26: 54.552$ & $+33: 33: 56.38$ & 25.8 & $2006 \mathrm{Jan}$ & $12: 26: 54.508$ & $+33: 33: 56.17$ & 20.5 & Spiral & $0.59(\mathrm{GYS})$ & Foreground \\
\hline CLJ1226.9+3332-cand2 & $12: 26: 55.503$ & $+33: 32: 12.42$ & 23.7 & 2003 Apr & $12: 26: 55.588$ & $+33: 32: 12.78$ & 20.2 & So & $0.9009(\mathrm{E})$ & Possible cluster SN Ia \\
\hline MS1054.4-0321-cand1 & $10: 56: 56.262$ & $-03: 37: 51.04$ & $24.9(i)$ & $2002 \mathrm{Dec}$ & 10:56:56.287 & $-03: 37: 51.59$ & 19.8 (i) & Spiral & 0.230 (FF5) & Foreground \\
\hline MS1054.4-0321-cand2 & $10: 56: 57.862$ & $-03: 37: 47.77$ & $23.5(i)$ & $2002 \mathrm{Dec}$ & $10: 56: 57.862$ & $-03: 37: 47.77$ & 21.3 (i) & So & $0.8335(\mathrm{VD})$ & Cluster SN Ia \\
\hline MS0451.6-0305-cand1 & 04:54:09.968 & $-03: 00: 28.18$ & 25.5 & $2005 \mathrm{Jul}$ & 04:54:10.054 & $-03: 00: 27.76$ & 18.3 & Spiral & 0.16 (FF3a) & Foreground \\
\hline
\end{tabular}


Table 2

Continued)

\begin{tabular}{|c|c|c|c|c|c|c|c|c|c|c|}
\hline \multicolumn{4}{|c|}{ Source } & \multirow{2}{*}{$\begin{array}{c}\text { Discovery } \\
\text { Epoch }\end{array}$} & \multicolumn{5}{|c|}{ Host } & \multirow[t]{2}{*}{ Classification } \\
\hline Name & R.A. (h m s) & Decl. $\left({ }^{\circ} \prime \prime \prime\right)$ & $(\mathrm{mag})^{\mathrm{b}}$ & & R.A. (h m s) & Decl. $\left({ }^{\circ} \prime \prime \prime\right)$ & $(\mathrm{mag})$ & Morphology & $\operatorname{Spec} z(\operatorname{Ref})^{c}$ & \\
\hline$\overline{\text { CL0152-1357-cand1 }}$ & 01:52:43.099 & $-13: 55: 19.76$ & $24.3(i)$ & 2005 Jun & 01:52:43.099 & $-13: 55: 19.76$ & 23.2 (i) & Compact & $1.27(\mathrm{GY})$ & Background AGN \\
\hline CL0152-1357-cand2 & 01:52:37.99 & $-13: 56: 25.69$ & $25.2(i)$ & 2005 Jun & 01:52:38.06 & $-13: 56: 25.5$ & 21.5 (i) & Spiral & 1.12 (FS) & Background \\
\hline CL0152-1357-cand3 & 01:52:46.217 & $-13: 58: 03.93$ & $24.1(i)$ & $2006 \mathrm{Sep}$ & $01: 52: 46.217$ & $-13: 58: 03.93$ & 23.9 (i) & Unclear & 0.839 (FS) & Cluster SN Ia \\
\hline SDSSJ1004+4112-cand1 & 10:04:33.086 & $+41: 12: 31.20$ & 25.2 & 2005 Dec & 10:04:33.075 & $+41: 12: 30.34$ & 22.3 & Spiral & $0.753(\mathrm{FF} 3 \mathrm{~b})$ & Background \\
\hline SDSSJ1004+4112-cand2 & 10:04:30.601 & $+41: 14: 10.66$ & 25.9 & $2004 \mathrm{Dec}$ & 10:04:30.645 & $+41: 14: 10.67$ & & $\ldots$ & $\cdots($ FF3b) & Possible cluster SN Ia \\
\hline SDSSJ1004+4112-cand3 & 10:04:31.007 & $+41: 14: 13.59$ & 26.3 & 2007 Jan & 10:04:31.047 & $+41: 14: 13.10$ & 21.6 & $\mathrm{E}$ & $0.75(\mathrm{O} 7)$ & Background \\
\hline
\end{tabular}

Notes.

${ }^{a}$ Coordinates are J2000.

b Magnitudes are observed $I$ band, unless indicated.

${ }^{c}$ Redshifts are based on the following observations: (FF1) Keck/LRIS 1" longslit, Filippenko and Foley, 2005 December 3; (FF2) Keck/DEIMOS 0'9 longslit, Filippenko and Foley, 2005 December 1; (FF3) Keck/DEIMOS (a) 0'9 longslit, (b) multislit, Filippenko and Foley, 2005 December 31; (FF4) Keck/LRIS 1" longslit, Filippenko and Foley, 2006 December 20; (FF5) Keck/DEIMOS multislit, Filippenko and Foley, 2007 February 16; (FF6) Keck/LRIS multislit, Filippenko and Foley, 2007 January 12; (FS) Keck/LRIS 1" longslit, Filippenko and Silverman, 2007 November 12; (FSP) Keck/LRIS

1" longslit, Filippenko, Silverman, and Poznanski, 2008 April 26. (GY) Keck/LRIS, 1" longslit, Gal-Yam, 2005 August 1; (VD) Keck/LRIS, 1"22 longslit, Van Dokkum et al. (2000); (E) Keck/DEIMOS, multislit, Ebeling, 2006 January; (GYS) Keck/LRIS multislit, Gal-Yam and Sharon, 2007 July 16-17; (MS) Keck/LRIS, 1" longslit, M. Sullivan, 2006 November 22; (O7) Keck/LRIS multislit, Ofek, 2007 January 22; (O8) Keck/LRIS multislit, Ofek, 2008 January 4. 


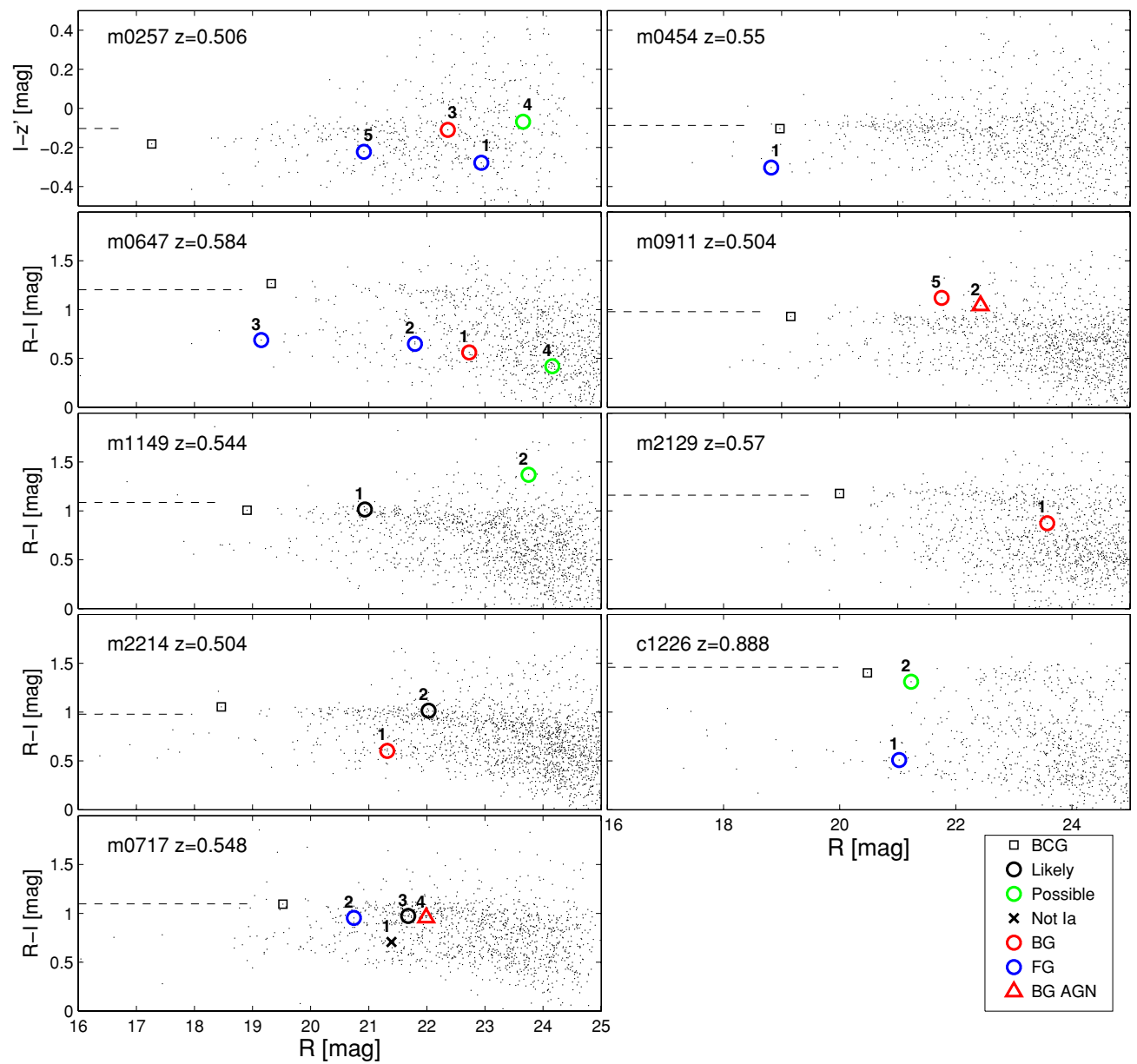

Figure 4. Color-magnitude diagrams for the galaxies in the cluster fields with SN candidates for which we have Subaru data. In each plot, the brightest cluster galaxy is marked with a black square. A dashed line indicates the color of an elliptical galaxy at the cluster redshift. Host galaxies are marked with circles (see the legend).

(A color version of this figure is available in the online journal.)

where available. In cases where the morphological type is unclear, we compare the location of the host in color-magnitude space with that of the cluster red sequence (Figures 4 and 5). For the MACS clusters, we use photometric catalogs of multiband Subaru images (see Section 3 for details). Multiband archival $H S T$ /ACS data are also available for some of the clusters. The classification of each individual candidate is described below.

\subsection{Candidates with Cluster-Member Hosts}

Likely cluster SNe Ia. Based on ACS morphology, colors, and Subaru photometry, four of the cluster candidate hosts are undoubtedly early-type galaxies: MACS0717 cand3, MACS1149 cand1, MACS2214 cand2, and MS1054 cand2. In the three cases where the candidate is not clearly separated from the galaxy core, we confirmed via spectroscopy that it is not an AGN. We therefore classify these events as likely cluster SNe Ia.

CLO152 cand3, likely cluster SN Ia. This candidate is detected in the second epoch with $I \approx 24.1 \mathrm{mag}$, slightly offset from the center of a cluster galaxy. Although the galaxy is bluer than the cluster red sequence, at $z=0.8391$ the $\mathrm{SN}$ absolute magnitude, $M_{B} \approx-18.4$, is strongly suggestive of an SN Ia, given the scarcity of overluminous CC SNe (less than $10 \%$ of CC SNe at maximum brightness are as bright in a volume-limited sample; W. Li et al. 2010, in preparation).

MACS0717 candl. The SN candidate appears in the archival epoch, in a spiral galaxy at the cluster redshift, and was observed in two filters, $V$ and $I$. The observed $V-I$ color $(\sim 1.6 \mathrm{mag})$ and magnitude $\left(I \approx 26.3\right.$, corresponding to $M_{V} \approx-15.3 \mathrm{mag}$ at $z=0.548$ ) are consistent with a CC SN. Young SNe Ia would be too bright (expected to be $<23 \mathrm{mag}$ ), and older SNe Ia would be too red. We therefore argue that this candidate is not an SN Ia.

\subsection{Candidates with Ambiguous Hosts}

CL1226 cand 2 appears in the archival epoch, 1". 12 from the core of an S0 galaxy at the cluster redshift. The $V-I$ color $(\sim 2.1 \mathrm{mag})$ and magnitude $(I \approx 23.7 \mathrm{mag})$ are consistent with an SN Ia, 9-10 days after maximum brightness. However, the candidate is also positioned close to a blue emission knot that is possibly a part of a disrupted spiral galaxy at unknown redshift, centered $\sim 5^{\prime \prime} \mathrm{NNW}$ of the cluster member. For SDSS1004 cand2, the results of the spectroscopy are ambiguous, with indications that the SN may be in a cluster-member galaxy. However, it is also on the outskirts of a foreground spiral galaxy at $z=0.27$. Adopting a conservative approach, we therefore classify these candidates as "Possible" cluster SNe Ia.

\subsection{Hostless Candidates}

Two of the SN candidates, MACS0911 cand 4 and MACS2129 cand2, have no detectable host at the limiting magnitude of the co-added ACS images. If these are indeed cluster events, they occurred $30 \mathrm{kpc}$ and $20 \mathrm{kpc}$ away from any galaxy, respectively. 

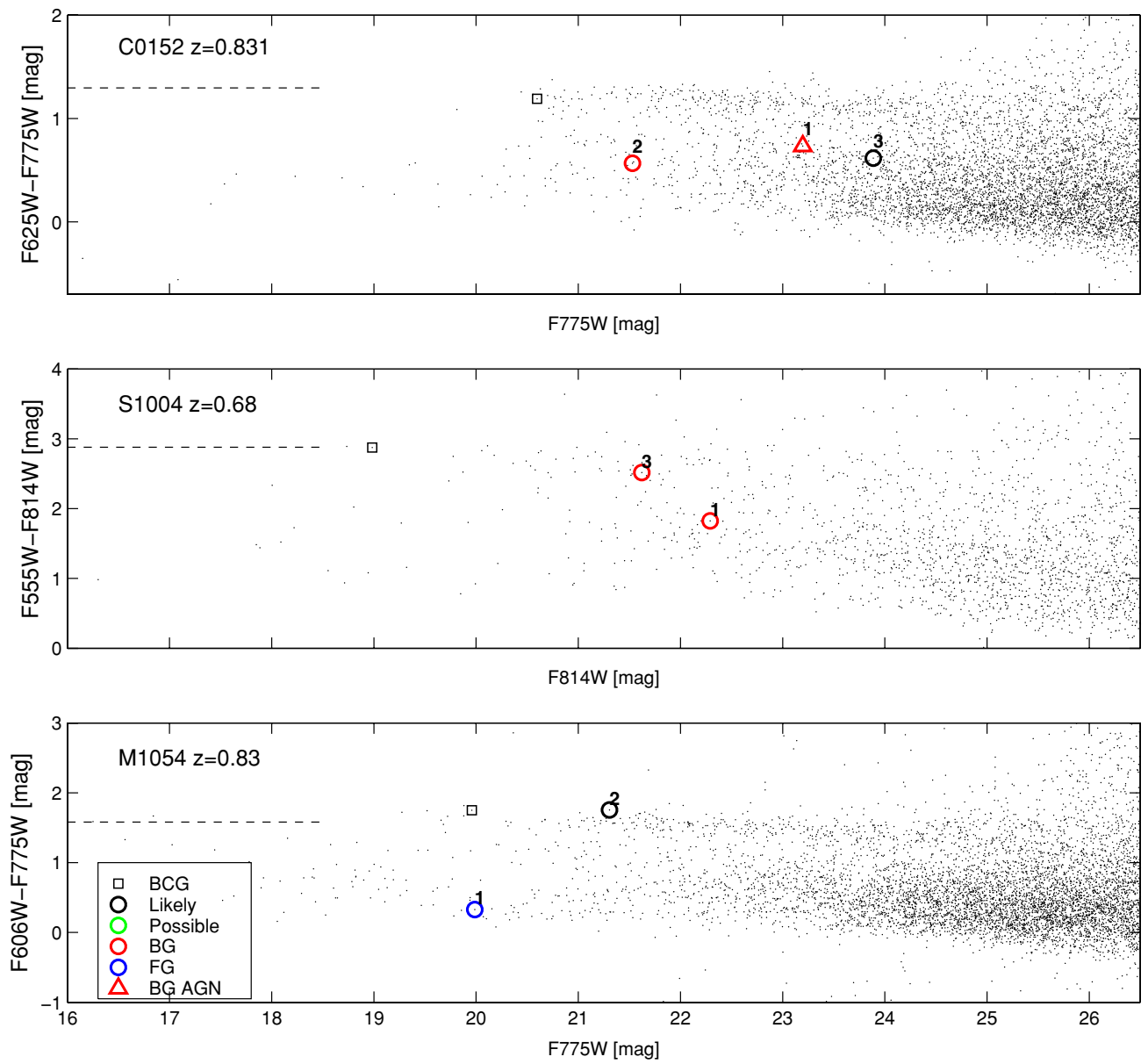

Figure 5. Same as Figure 4, but for cluster fields for which we do not have Subaru data. The magnitudes are measured in the available ACS bands in the archive. (A color version of this figure is available in the online journal.)

The absolute magnitudes of these candidates, $M_{V}=-16.4$ and $M_{V}=-17.5$, are consistent with SNe Ia at these clusters' redshifts. Their projected distances from the brightest cluster galaxies (BCGs) are $0.49 \mathrm{Mpc}$ and $0.38 \mathrm{Mpc}$, respectively, at the clusters' redshifts. The $V-I$ color of MACS0911 cand4 does not rule out any SN type at the cluster redshift. These, if they are intergalactic cluster events (Gal-Yam et al. 2003), are most probably $\mathrm{SNe}$ Ia, since intergalactic $\mathrm{CC}$ SNe should be very rare; there is little or no star formation in the intergalactic medium (Gal-Yam et al. 2003), and even if a CC SN progenitor is ejected from its host galaxy, it cannot move far before exploding since it is short lived. The progenitors of SNe Ia, on the other hand, have ample time to reach $30-70 \mathrm{kpc}$ from their host galaxy before explosion.

\subsection{Candidates Without Measured Host Redshift}

We do not have secure redshift measurements for four of the SN candidate hosts. Spectroscopy was attempted (unless indicated), but the objects proved to be too faint and/or without emission lines. The host of MACS0257 cand2 is too faint for spectroscopy, with $I \gtrsim 27 \mathrm{mag}$. The luminosity of the SN candidate does not rule it out as an SN Ia. MACS0257 cand4 is offset from the core of a resolved host with an elliptical morphology. The host colors are consistent with the cluster red sequence in the available Subaru bands $\left(I, z^{\prime}\right)$. MACS1149 cand 2 is offset from the core of a host with an early-type morphology. The host colors are consistent with the red sequence in $V-I$, but are redder than cluster galaxies in $R-I$ and $R-z^{\prime}$. Spectroscopy of this galaxy failed due to a mistake in mask designs, as a result of which the galaxy spectrum fell off the CCD. MACS0647 cand4 is offset from the core of a resolved host with unclear morphological type. Subaru colors indicate that it is $\sim 1.5$ mag bluer than the cluster red sequence.

\subsection{Classification Summary}

We conclude that five of the cluster events are likely SNe Ia, and one is likely a CC SN. To these we add, as possible cluster $\mathrm{SNe}$ Ia, the two hostless $\mathrm{SNe}$, the two SNe with ambiguous hosts, and the four candidates for which we do not have measured redshifts. In terms of radial distribution, the cluster $\mathrm{SNe}$ are found at projected distances of up to $0.7 \mathrm{Mpc}$ from the $\mathrm{BCG}$ (see Table 4).

In order to reduce our uncertainties due to misclassification, when calculating the SN Ia rate we impose a magnitude cut and remove from our sample candidates fainter than $26 \mathrm{mag}$. This changes the SN count to five likely and six possible cluster SNe Ia.

\section{SN RATE CALCULATION}

With the sample of potential cluster SNe Ia constructed above, we can now derive an $\mathrm{SN}$ rate for our cluster sample. The SN 
Table 3

Subaru Imaging Data

\begin{tabular}{|c|c|c|c|c|c|c|c|c|c|c|}
\hline \multirow[t]{2}{*}{ Cluster Name $^{a}$} & \multicolumn{5}{|c|}{ UT Date Observed (mm/yy) } & \multicolumn{5}{|c|}{ Exposure time (s) } \\
\hline & $B$ & $V$ & $R$ & $I$ & $z^{\prime}$ & $B$ & $V$ & $R$ & $I$ & $z^{\prime}$ \\
\hline MACSJ0257 & $09 / 05$ & $12 / 02$ & $12 / 00$ & $12 / 07$ & $12 / 02$ & 1440 & 2160 & 5280 & 2400 & 2700 \\
\hline MACSJ0454 ${ }^{\mathrm{b}}$ & $11 / 05$ & $11 / 05$ & $03 / 05$ & $12 / 01$ & $12 / 06$ & 1440 & 2160 & 3240 & 2160 & 1440 \\
\hline MACSJ0647 & $11 / 05$ & $09 / 03$ & $02 / 04$ & $02 / 04$ & $02 / 04$ & 1440 & 2160 & 2880 & 2880 & 2160 \\
\hline MACSJ0717 & $02 / 04$ & $12 / 02$ & $12 / 00$ & $12 / 00$ & $12 / 02$ & 1440 & 2160 & 2880 & 1440 & 1620 \\
\hline MACSJ0744 & $02 / 04$ & $04 / 03$ & $12 / 02$ & $04 / 03$ & $04 / 03$ & 1440 & 1440 & 2880 & 3240 & 2160 \\
\hline MACSJ0911 & $11 / 05$ & $04 / 03$ & $04 / 03$ & $04 / 03$ & $04 / 03$ & 2880 & 2160 & 2880 & 1200 & 1620 \\
\hline MACSJ1149 & $12 / 06$ & $04 / 03$ & $04 / 03$ & $12 / 00$ & $04 / 03$ & 1440 & 2160 & 2880 & 1200 & 1620 \\
\hline MACSJ1423 & $07 / 03$ & $06 / 02$ & $06 / 02$ & $06 / 02$ & $06 / 02$ & 1920 & 2160 & 2400 & 2160 & 1440 \\
\hline MACSJ2129 & $07 / 03$ & $06 / 02$ & $06 / 01$ & $06 / 01$ & $06 / 02$ & 2880 & 1440 & 2880 & 2880 & 1440 \\
\hline MACSJ2214 & $11 / 05$ & $09 / 03$ & $07 / 03$ & $07 / 04$ & $07 / 04$ & 1440 & 2160 & 2880 & 2160 & 1620 \\
\hline CL1226 & $05 / 06$ & $06 / 02$ & $12 / 00$ & $12 / 00$ & $04 / 03$ & 2160 & 2160 & 2880 & 1920 & 1080 \\
\hline
\end{tabular}

Notes.

${ }^{\text {a }}$ Full cluster names are listed in Table 1.

b MACSJ0454.1-0300 is also named MS0451.6-0305.

rate per unit stellar luminosity is calculated as follows:

$$
R_{\mathrm{Ia}}=\frac{N}{\sum_{j} \Delta t_{j} L_{\mathrm{band}, j}},
$$

where $N$ is the number of $\mathrm{SNe}, \Delta t_{j}$ is the visibility time (or "control time," the time during which a cluster SN Ia is above the detection limit of the $j$ th image), $L_{\text {band,j }}$ is the cluster luminosity within the search area of the $j$ th image in the chosen photometric band, and the summation is over all the survey images. The details of each element in the calculation are given below. To account for the statistical nature of some of the quantities that enter the rate calculation, we conduct a Monte Carlo simulation in which we measure the rate many times, each time assigning values to the measured quantities by drawing them from a distribution (see Section 7.3). The final rate and its uncertainty is measured from a histogram of the results.

\subsection{Visibility Time}

The visibility time depends on the detection efficiency, the peak luminosity, and the shape of the light curve at a given redshift and filter. In principle, it can also depend on the time interval between observations, as an SN is less likely to be detected via subtraction if it has a similar brightness at both epochs. In practice, since all of our comparisons are for epochs that are separated by at least one year, old $\mathrm{SNe}$ would have had enough time to decline below our detection limit, and thus the visibility time is not affected. We calculate the effective visibility time from

$$
\Delta t_{j}=\int_{-\infty}^{\infty} \eta[m(t)] d t
$$

where $m(t)$ is the SN Ia light curve in the image bandpass (either F814W or F775W) at the given redshift and $\eta[m(t)]$ is the detection probability as a function of SN magnitude. We describe below each step in this calculation.

\subsubsection{Detection Efficiency Estimate}

To determine the detection efficiency of our survey, we conducted efficiency simulations following the scheme detailed by Gal-Yam et al. (2002) and Sharon et al. (2007). To each field, we added some 200 fake $\mathrm{SNe}$, in a range of magnitudes, and with a spatial distribution that follows the flux of the galaxies. The simulated images then underwent the same search procedure as the real data, and the number of $\mathrm{SNe}$ that were recovered in each magnitude bin was noted. We find that while the efficiency function strongly depends on the limiting magnitude of the image, its shape is also sensitive to other attributes of the field and of the observation. Although HST images do not suffer from atmospheric distortion of the PSF, the cores of bright galaxies are not perfectly subtracted in difference images, resulting in residuals that can hide faint transients. This effect is amplified when two epochs are not obtained at the same position angle due to position-dependent variations in the PSF. We experimented with several PSF-matching techniques (e.g., Gal-Yam et al. 2008) and determined that a simple subtraction is sufficient for our purposes. We find that there is $100 \%$ efficiency in all epochs to detect $\mathrm{SNe}$ at magnitudes brighter than 23 , even in the cores of bright galaxies. The efficiency differs from field to field, and even between epochs of the same field. Figure 6 shows the results of our efficiency simulations. We parameterized our efficiency curves as a function of magnitude $m$ with the function

$$
\eta\left(m ; m_{0.5}, s, s_{2}\right)= \begin{cases}\left(1+e^{\frac{m-m_{0.5}}{s}}\right)^{-1}, & m \leqslant m_{0.5} \\ \left(1+e^{\frac{m-m_{0.5}}{s_{2}}}\right)^{-1}, & m>m_{0.5},\end{cases}
$$

where $m_{0.5}, s$, and $s_{2}$ are free parameters that are fit to the simulated efficiencies: $m_{0.5}$ is the magnitude at which the efficiency drops to 0.5 , and $s$ and $s_{2}$ determine the range of $m$ over which $\eta$ changes from 1 to 0.5 and from 0.5 to zero, respectively.

In the final rate calculation, we apply a magnitude cut at 26 mag by setting the efficiency above 26 mag to zero, and rejecting from the sample $\mathrm{SNe}$ that were detected above that value.

\subsubsection{Light Curves}

The light curves of SNe Ia exhibit an empirical inhomogeneity, where luminous SNe tend to rise and decline more slowly than subluminous ones. This means that luminous $\mathrm{SNe}$ will be above the detection limit longer than subluminous ones, resulting in an overall visibility time that is correlated with SN brightness. The correlation between peak magnitude and lightcurve shape can be parameterized by a stretch relation (Phillips 


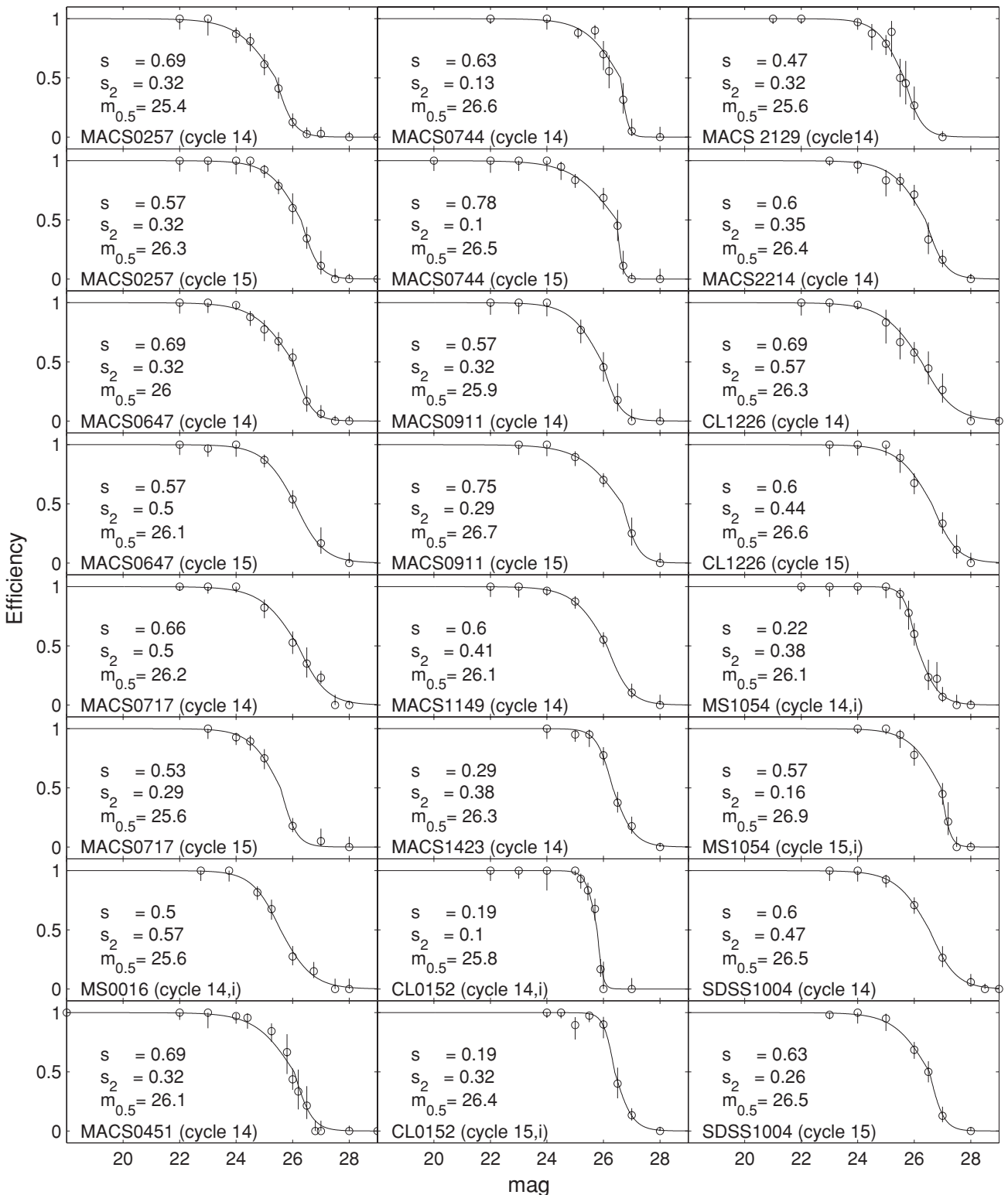

Figure 6. Detection efficiency curves for a point source in a difference image, as a function of magnitude. The difference images are epoch II vs. epoch I (indicated by "cycle 14") and epoch III vs. epoch II (indicated by "cycle 15"). Magnitudes are measured in the difference image, and are Vega-based I-band magnitudes, except where indicated by "i." Cluster names are as marked. Circles mark the fraction of detected fake SNe, with error bars based on a binomial distribution. Solid lines are the best-fit efficiency curves with the indicated parameter values, parameterized as in Equation (3).

1993; Perlmutter et al. 1999; see Leibundgut 2001 for a review), which exhibits an intrinsic root mean square (rms) scatter of $\sim 0.2 \mathrm{mag}$. In this paper, we use the form $M_{s}=M_{s=1}-\alpha(s-1)$, $t_{s}=t_{s=1} \times s$, as described by Perlmutter et al. (1999), where $s$ is the stretch factor ( $s=1$ means an unstretched light curve), $M_{s}$ is the absolute $B$-band magnitude, and $\alpha=1.47$, based on the empirical results of Knop et al. (2003). The peak-magnitude distribution of SNe Ia also depends on the host-galaxy properties. By comparing light curves of $\mathrm{SNe}$ in different environments, Sullivan et al. (2006b) have shown that SNe Ia in elliptical galaxies tend to be dimmer (with a smaller stretch factor) than those in star-forming galaxies (Hamuy et al. 2000; Howell 2001).

For each cluster, we compile a set of light curves relevant to the cluster redshift and imaging band for several lightcurve shapes and peak magnitudes. We start with a restframe, non-stretched, $B$-band template light curve from Nugent et al. (2002) and transform it to various stretched light curves using the stretch relation above and the measured distribution of elliptical host-galaxy stretch factors from Sullivan et al. (2006b). For consistency, we use the same non-stretched peak $B$-band absolute magnitude as Sullivan et al. (2006a), $M_{B}=$ $-19.25 \mathrm{mag}$ (for $H_{0}=70 \mathrm{~km} \mathrm{~s}^{-1} \mathrm{Mpc}^{-1}$ ). We assume an uncertainty of $0.15 \mathrm{mag}$ in $M_{B}$, from the dispersion in peak magnitudes of local SN Ia light curves after applying a stretch correction (Guy et al. 2005). This uncertainty is taken into account in our error budget (see Section 7.3).

A set of multi-epoch spectral templates from Nugent et al. (2002) are normalized to fit the $B$-band rest-frame stretched light curve, and redshifted according to the cluster redshift. We then combine the redshifted spectra with the HST bandpass in which the cluster was observed, to obtain a light curve for each combination of stretch, redshift, and bandpass in our survey. When calculating the visibility time for a particular image, we draw for each SN in the Monte Carlo simulation a stretch factor 
(with its corresponding properly normalized light curve) from the Sullivan et al. (2006b) distribution of stretch factors. Table 1 lists the visibility time for each epoch for the most likely stretch factor. We note that since epochs I and II are searched against each other, the values enumerated in Table 1 (i.e., visibility time, $L, M$, and the search area) are the same for epochs I and II and are only listed once.

\subsection{Cluster Stellar Luminosity}

An $\mathrm{SN}$ rate in a targeted galaxy population needs to be normalized by the stellar luminosity or mass of that population. These stellar properties, in turn, must be measured to some level of accuracy. Our rate measurement accuracy is limited by the small number of $\mathrm{SNe}$ that were discovered, with a $1 \sigma$ statistical error of order 30\%-40\% (lower and upper Poisson errors for 10 events; see Section 7.3.1). An accuracy of $10 \%$ in the cluster stellar luminosities will therefore be more than satisfactory for our purposes. We generally follow the route detailed by Sharon et al. (2007), and perform "aperture photometry" of the cluster light in several bands, as follows. For the MACS clusters and CLJ1226.9+3332, we use the Subaru data centered on each cluster (see Section 3). For most of the MACS clusters, we were able to use calibrated $V, R$, and $I$ images, with additional $B$ - and $z^{\prime}$-band imaging for some.

Using the galaxy catalogs described in Section 3, we measure the total flux in galaxies within a given aperture and subtract from it the flux per unit area in galaxies in a "background" area at the outskirts of the images, multiplied by the area of the aperture. This way, we statistically subtract the contribution of "background" objects (those in front of and behind the cluster). The aperture in which we measure the cluster stellar luminosity is the exact search area (i.e., the area of overlap between epochs). The "background" is sampled in an annulus with area of at least $100 \operatorname{arcmin}^{2}$, with an inner radius of at least $7^{\prime}$ from the cluster center. These "background" annulus radii vary among the sample clusters, depending on the angular size of the cluster and the available Subaru field of view, and take into account masking of bright foreground objects. The completeness of the Subaru data varies between clusters and depends on exposure time and observational conditions. For each cluster, we estimate the completeness magnitude as the turnover magnitude in its galaxy-magnitude histogram in the $R$ band. We ignore in our calculation galaxies fainter than the completeness magnitude, and correct the total luminosity accordingly as explained below. To avoid contamination from bright low-redshift objects, we also ignore galaxies that are brighter than the BCG.

The luminosities of the four clusters for which we do not have Subaru data were measured in the same manner, but from the HST data. Since the ACS field of view is too small to select "background" annuli around the clusters, we assumed a universal background, drawn from the archival HST/ACS imaging of The Great Observatories Origins Deep Survey (GOODS; Giavalisco et al. 2004).

For comparison with other SN rate measurements, we convert the observed net integrated galaxy-light fluxes in the available bands to rest-frame luminosities in several bands and form a cluster spectral energy distribution (SED). To each cluster SED, we fit redshifted template spectra of combinations of several types of galaxies using synthetic photometry. For elliptical galaxies, we assume the Bruzual \& Charlot (2003) synthesis of stellar population with a single formation epoch at $z=3$. Other galaxy types are represented by templates from Kinney et al. (1996). Typically, within each search area (i.e., the innermost $\sim 1 \mathrm{Mpc}$ of each cluster in our survey), the best fit was reached for a combination of 70\%-80\% elliptical-galaxy flux and 20\%-30\% Sbc-galaxy flux. The fact that there is a nonnegligible fraction of blue galaxies is consistent with recent measurements of the red fraction of galaxies in clusters in the redshift range of our survey (Loh et al. 2008) due to the Butcher $\&$ Oemler $(1978,1984)$ effect. The luminosities in the desired rest-frame bands are measured via synthetic photometry of the best-fit template combination, scaled to fit the observed net cluster flux.

To account for undetected cluster galaxies, we correct the luminosity by multiplying it by the fraction of light that comes from the faint end of a Schechter (1976) luminosity function

$$
C=\frac{\int_{0}^{\infty} L \Phi(L) d L}{\int_{L_{\lim }\left(m_{\lim }, z\right)}^{\infty} L \Phi(L) d L},
$$

where $\Phi(L) d L=\Phi^{*}\left(L / L^{*}\right)^{\alpha} \exp \left(-L / L^{*}\right) d\left(L / L^{*}\right)$. We adopt $\alpha=-1.00 \pm 0.06$ and $M^{*}=-22.01 \pm 0.11 \mathrm{mag}$ as the mean values for the $g$-band luminosity function parameters in clusters (Goto et al. 2002). We note that the depth of the Subaru images enables detection of galaxies down to $M_{I}=-17 \mathrm{mag}$, $\sim 5$ mag fainter than $M^{*}$, and thus the necessary correction is small, typically $1 \%-5 \%$ for the lower-redshift clusters, and up to $\sim 11 \%$ for the clusters at $z>0.8$.

Finally, to account for passive evolution of the dominant elliptical-galaxy component over the redshift range of the cluster sample, the Bruzual \& Charlot (2003) component is passively evolved backward or forward, as appropriate, to the mean visibility-weighted redshift of the sample, $\langle z\rangle=0.6$ (see Table 1). The Sbc component is not evolved in this calculation. This correction amounts to a $2 \%$ change in the total luminosity.

\subsection{Error Budget}

In this section, we estimate the sources of uncertainty, both statistical and systematic. While the statistical errors can be propagated in a straightforward manner, the systematic errors affect the final result in a more complicated way, and may be correlated. To assess the overall systematic uncertainty, we calculate the $\mathrm{SN}$ rate by performing a Monte Carlo simulation in which we measure the rate many times, each time assigning values to the measured quantities by drawing them from a distribution centered on the best value, with a width according to the uncertainty of this value. Where applicable, we draw the values from a measured distribution and otherwise assume a Gaussian distribution.

\subsubsection{Statistical Errors}

The counting of SN explosions obeys Poisson statistics, from which we derive the statistical uncertainties. Contrary to the results presented for $z \approx 0.2$ clusters by Sharon et al. (2007), the number of secure cluster SNe in the present survey is not certain, due to the uncertainty in $\mathrm{SN}$ classification and redshift, and is between 5 and 11. We will consider this classification uncertainty as a systematic error below. If we adopt for the central number of cluster SNe Ia the mean in this range, 8 , the $1 \sigma$ Poisson errors are $+49 \%,-35 \%$ (Gehrels 1986).

\subsubsection{Systematic Errors}

SN classification uncertainties. A significant source of error in our rate derivation is the possible misclassification of SNe. Since our sample is not spectroscopically confirmed, some of 
Table 4

Cluster-member SN Candidates

\begin{tabular}{|c|c|c|c|c|c|}
\hline Name & $M_{V}^{\mathrm{a}}$ & $M_{B}^{\mathrm{b}}$ & $R^{\mathrm{b}}$ & Classification & Note \\
\hline MACSJ0257-2325-cand2 & -14.7 & $\ldots$ & 0.48 & Possible & Unknown $z$ \\
\hline MACSJ0257-2325-cand4 & -15.0 & $\ldots$ & 0.51 & Possible & Unknown $z$, early red-sequence host ${ }^{\mathrm{c}}$ \\
\hline MACSJ0647+7015-cand4 & -16.6 & $\ldots$ & 0.37 & Possible & Unknown $z$ \\
\hline MACSJ0717+3745-cand1 & -15.3 & $\ldots$ & 0.56 & Not SN Ia & Cluster $z$, likely CC SN \\
\hline MACSJ0717+3745-cand3 & -17.4 & $\ldots$ & 0.69 & Likely & Cluster $z$, early host \\
\hline MACSJ0911+1746-cand4 & -16.4 & $\ldots$ & 0.49 & Possible & Hostless \\
\hline MACSJ1149+2223-cand1 & -17.8 & $\ldots$ & 0.32 & Likely & Cluster $z$, early host \\
\hline MACSJ1149+2223-cand2 & -17.2 & $\ldots$ & 0.60 & Possible & Unknown $z$, early host \\
\hline MACSJ2129-0741-cand2 & -17.5 & $\ldots$ & 0.38 & Possible & Hostless \\
\hline MACSJ2214-1359-cand2 & -17.8 & $\ldots$ & 0.02 & Likely & Cluster $z$, early host \\
\hline CLJ1226.9+3332-cand2 & $\ldots$ & -18.6 & 0.38 & Likely & Cluster $z$, early host \\
\hline MS1054.4-0321-cand2 & $\ldots$ & -18.9 & 0.26 & Likely & Cluster $z$, early host \\
\hline CL0152-1357-cand3 & $\ldots$ & -18.4 & 0.44 & Likely & Cluster $z$, brightness suggests SN Ia \\
\hline SDSSJ1004+4112-cand2 & -15.8 & $\ldots$ & 0.67 & Possible & One of the possible hosts is at cluster $z$ \\
\hline
\end{tabular}

Notes.

a Absolute magnitude at the time of detection, $K$-corrected to rest-frame $V$ for candidates at $z<0.7$ and to rest-frame $B$ for candidates at $z>0.7$. In cases of unknown redshift, the SN candidate is assumed to be at the cluster redshift.

${ }^{\mathrm{b}}$ Projected distance from brightest cluster galaxy, in Mpc.

c The host has the same $i-z^{\prime}$ color as the cluster red-sequence galaxies; see Figure 4.

the SN candidates may possibly be $\mathrm{CC}$ SNe at the cluster redshifts. CC SNe are preferentially located in star-forming regions, and are rarely found in the elliptical galaxies that dominate galaxy cluster environments. Although some cluster star formation may still be in progress, especially in high-redshift clusters, Saintonge et al. (2008) find that such activity tends to be outside the central $1 \mathrm{Mpc}$, while all of our cluster candidate $\mathrm{SNe}$ are within $0.7 \mathrm{Mpc}$ in projection. Nevertheless, while many of the cluster SN candidates in our sample occurred in elliptical galaxies, we note that some are associated with galaxies that have a late-type morphology. We will therefore conservatively allow for a maximal misclassification error.

As described in detail in Section 6, we set a firm lower limit on the number of detected cluster SNe Ia from the five candidates in early-type cluster galaxies. Eight candidates are considered possible cluster SNe Ia, of which two are fainter than 26 mag and are rejected from our sample. This sets the upper limit due to the classification uncertainty at 11 .

Luminosity error. The derived cluster stellar luminosities depend on several assumptions, such as the choice of area from which to draw the background, photometric errors, and the assumption of the galaxy templates with which the cluster SED was converted to rest-frame luminosity. To account for these uncertainties, we perform a Monte Carlo simulation, similar to those presented by Sharon et al. (2007). To assess the uncertainty introduced by our choice of background area, we explored different choices of backgrounds for each cluster. We find that for a $\lesssim 10 \%$ variation in the inner radius of the background annulus, the measured luminosity changes by $\lesssim 5 \%$. Skewing our measurement of the fraction of elliptical light in the cluster by 0.1 results in a $\lesssim 10 \%$ change in the measured luminosity. Assigning a $10 \%$ uncertainty to the luminosity of individual clusters in the Monte Carlo simulation results in a $3.4 \% \mathrm{rms}$ variation of the $\mathrm{SN}$ rate.

Efficiency error. As explained in Section 7.1.1, we represent each efficiency curve by a continuous function that we fit to the results of efficiency simulations. To assess the errors induced by this process, and by the fact that the number of fake SNe from which the efficiency is derived is finite, we consider a range of efficiency-function parameters distributed normally around the best-fit value, with a $10 \%$ dispersion. In each realization in the Monte Carlo simulation, we draw a set of parameters from this distribution, resulting in an uncertainty due to the efficiency estimation of $1.6 \%$.

Visibility time error. The visibility time depends on the shapes and peak magnitudes of the SN light curves at the cluster redshifts. These values are correlated through the stretch relation (see Section 7.1.2). We explore a range of stretch parameters, following the distribution published by Sullivan et al. (2006b). In each realization in the Monte Carlo simulation, we draw a stretch factor from this distribution and assign a light curve accordingly. Since the visibility time is not linear with a change of the stretch factor, the resulting distribution of $\mathrm{SN}$ rates is no longer centered on the value that is calculated from the most probable stretch factor. The inferred uncertainty due to the visibility time error is $2.7 \%$.

Accounting for all of the above systematic errors in the Monte Carlo simulation, we get an overall systematic uncertainty of $5 \%$. This uncertainty is, at present, negligible compared to the statistical errors, but will become relevant in future surveys that detect more than several hundreds of SNe.

\subsection{Results}

The visibility-time-weighted mean redshift of our cluster sample is $\langle z\rangle=0.6$. For the adopted central value of $N_{\text {Ia }}=8$, our measured SN rate per unit $B$-band stellar luminosity for this cluster sample is $0.35 \mathrm{SNu}_{B}{ }_{-0.12}^{+0.17}$ (statistical) \pm 0.13 (classification) \pm 0.01 (systematic).

The SN rate per unit luminosity at $\langle z\rangle=0.6$ cannot be compared easily to rates at low redshifts even in the absence of any star formation because of significant passive luminosity evolution. For example, between $z=0.6$ and $z=0$, a passive population fades by about a factor of 2 in blue bands (Bell et al. 2003; van der Wel et al. 2005). To facilitate such a comparison, we also list in Table 5 a rate in units of $\mathrm{SNu}_{B, 0}$, which is a cluster $\mathrm{SN}$ rate at $\langle z\rangle=0.6$ per unit stellar luminosity, but after passively evolving forward, to $z=0$, the elliptical Bruzual \& Charlot (2003) component fit to each cluster's photometry (the 
Table 5

Cluster SN Ia Rate At $\langle z\rangle=0.6$

\begin{tabular}{lcccc}
\hline \hline Units $^{\mathrm{a}}$ & SN Rate & Statistical Error & Classification Error & Systematic Error \\
\hline $\mathrm{SNu}_{M}$ & 0.112 & +0.055 & \pm 0.042 & \pm 0.005 \\
$\mathrm{SNu}_{B}$ & 0.35 & -0.039 & \pm 0.13 & \pm 0.01 \\
$\mathrm{SNu}_{B, 0}$ & 0.68 & -0.17 & \pm 0.26 & \pm 0.03 \\
\hline
\end{tabular}

Note.

a $\mathrm{SNu}_{B}$ denotes $\mathrm{SNe}\left(100 \mathrm{yr} 10^{10} L_{B, \odot}\right)^{-1}$. $\mathrm{SNu}_{M}$ denotes $\mathrm{SNe}(100 \mathrm{yr}$ $\left.\left.10^{10} M_{\odot}\right)^{-1}\right]$, and $\mathrm{SNu}_{B, O}$ denotes a rate normalized to cluster luminosity that is passively evolved $z=0$.

blue component, which contributes negligibly to the mass, is ignored in this calculation).

To derive the rate normalized by stellar mass, we follow Mannucci et al. (2005), who converted $K$-band galaxy luminosities to mass using the mass-to-light ratio derived by Bell $\&$ de Jong (2001) and observed galaxy $B-K$ colors. We derive the rest-frame $g-r$ colors of each cluster from the best-fit combination of template spectra. The stellar masses of the red and blue components of each cluster are then estimated from the color-dependent stellar mass-to-light ratio derived by Bell et al. (2003), $\log _{10}\left(M_{\odot} / L_{g, \odot}\right)=-0.499+1.519(g-r)$ (see Mannucci et al. 2005 for a discussion of the validity of this ratio for our purpose). The total stellar mass in each search area is enumerated in Table 1. The SN rate is calculated from Equation (1), in which we replace the stellar luminosity with the inferred total mass within the search area of each cluster. For $N_{\mathrm{Ia}}=8$, the resulting $\mathrm{SN}$ Ia rate per unit stellar mass is $0.112 \mathrm{SNu}_{M}{ }_{-0.039}^{+0.055}$ (statistical) \pm 0.042 (classification) \pm 0.005 (systematic). The different rates derived in this work are listed in Table 5.

\section{DISCUSSION AND SUMMARY}

Our newly measured $\mathrm{SN}$ rate at $z \approx 0.6$ is the most accurate to date for clusters at such high redshifts. Combined with previous cluster-rate measurements, we are, for the first time, in a position of being able to examine the evolution of the $\mathrm{SN}$ rate in clusters over a greater part of cosmic history. Figure 7 shows the cluster $\mathrm{SN}$ rate as a function of redshift, at $\langle z\rangle=0.02$ (Mannucci et al. 2008), $\langle z\rangle=0.08$ and 0.22 (Dilday et al. 2010), $\langle z\rangle=0.15$ (Sharon et al. 2007), $\langle z\rangle=0.25$ and 0.90 (Gal-Yam et al. 2002), $\langle z\rangle=0.46$ (Graham et al. 2008), and $\langle z\rangle=0.6$ (this work). For consistency, the rates from Gal-Yam et al. (2002) were converted from $\mathrm{SNu}_{B}$ to $\mathrm{SNu}_{M}$ using the ratio found by Sharon et al. (2007) for the lower-redshift bin, and using the ratio found in this work for the higher-redshift bin. The SN rate in the redshift bin studied in this work is consistent with the lower-redshift rate measurements (to within uncertainties), and shows that there is, at most, only a slight increase of cluster SN rate with redshift.

In a companion paper (Maoz et al. 2010b), we analyze the results and discuss the implications of the observed rates on $\mathrm{SN}$ progenitor models and on the role of $\mathrm{SNe}$ in the metal enrichment of the ICM. However, some conclusions emerge directly from the low $\mathrm{SN}$ rate observed out to $z \approx 1$. Independent of any model, the integral over the observed SN Ia rate per unit mass between $z=1$ and 0 gives the total number of SNe per unit stellar mass. If multiplied further by the mean iron yield of an SN Ia, $0.7 M_{\odot}$ (e.g., Mazzali et al. 2007), one obtains $M_{\mathrm{Fe}} / M_{*}$, the ratio of the iron mass produced in clusters over that cosmic period to the present-day stellar mass. Assuming, for example, a constant SN Ia rate of $0.1 \mathrm{SNu}_{M}$ over the past $6 \mathrm{Gyr}$,

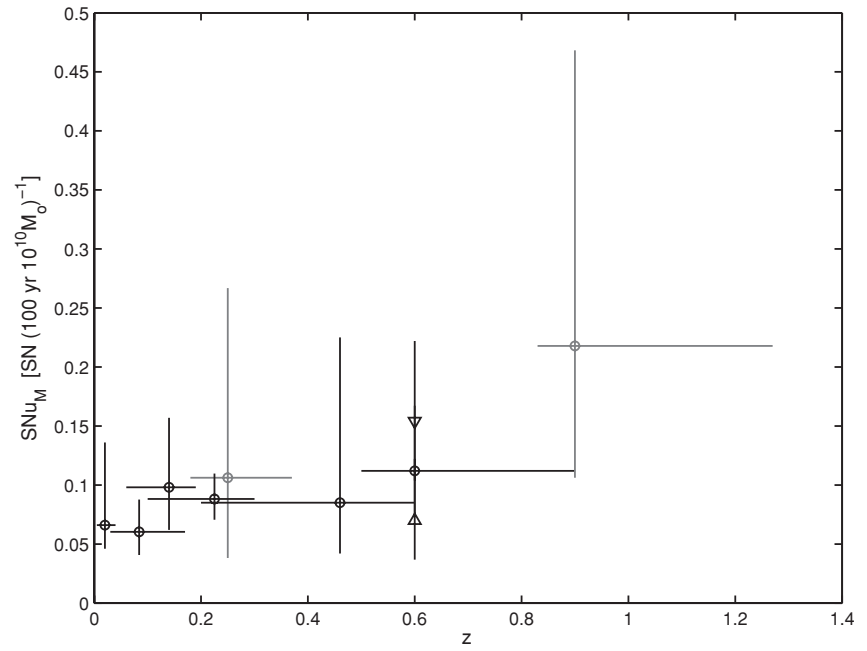

Figure 7. Cluster SN rates from (in order of increasing redshift) Mannucci et al. (2008), Dilday et al. (2010), Sharon et al. (2007), Dilday et al. (2010), Gal-Yam et al. (2002), Graham et al. (2008), this work, and Gal-Yam et al. (2002). Triangles represent the $\mathrm{SN}$ rate derived from the upper and lower limits on the observed number of cluster SNe Ia, 11 and 5, respectively. Vertical error bars are $1 \sigma$ uncertainties, and horizontal error bars show the cluster samples' redshift ranges.

as implied by Figure 7 , gives $M_{\mathrm{Fe}} / M_{*} \approx 0.0004$. In contrast, the observed ratio in present-day clusters, after subtracting the expected contribution from CC SNe, is $\left[M_{\mathrm{Fe}} / M_{*}\right]_{\mathrm{obs}} \approx 0.004$ (see compilation and analysis of Maoz 2008), an order of magnitude higher. Thus, only a small fraction, $\lesssim 10 \%$, of the iron mass could have been produced by SNe Ia between $z=1$ and 0 . This conclusion is strengthened if one assumes, instead of $0.7 M_{\odot}$, the lower iron yields associated with subluminous $\mathrm{SNe}$ Ia. Such SNe tend to occur in the early-type galaxies that dominate galaxy cluster cores. The factor of $\sim 2$ increase in ICM iron abundance between these two redshifts, recently reported by Balestra et al. (2007) and Maughan et al. (2008), cannot be the effect of new iron production during this time interval, and must instead be a redistribution effect. Alternatively, the abundance evolution may not be real (Ehlert \& Ulmer 2009), in which case the non-evolution is fully consistent with our SN results.

Our measurements constrain the fraction of intergalactic SNe Ia (Gal-Yam et al. 2003). Assuming the two hostless SNe are intergalactic cluster events, and that only the six most secure events in our sample are indeed $\mathrm{SNe}$ Ia in cluster galaxies, we get an upper limit on the relative fraction of intergalactic events of $2 / 8$. This is very similar to the fraction measured by Gal-Yam et al. (2003) in lower redshift $(z \approx 0.1)$ clusters $(2 / 7)$. Since we cannot rule out that the two hostless sources we found are not cluster SNe Ia (i.e., that they reside in faint background galaxies, or are not $\mathrm{SNe}$ at all), the lower limit on the intergalactic SN Ia fraction could be as low as zero. Assuming that $1 \pm 1$ of these events are cluster SNe Ia and that our cluster SN Ia sample includes both the six likely and seven possible events we listed above, we get a likely fraction of $1 / 13$ with an uncertainty of a factor of a few. This is several times below the value we measured at lower redshift (Gal-Yam et al. 2003), as would be expected from models (Dubinski 1998) which predict that the intergalactic population of stars is mostly assembled between $z=1$ and $z=0$.

To summarize, we have conducted a survey for SNe in the fields of 15 galaxy clusters, using new and archival HST/ACS 
data. Each cluster was visited two to three times, for at least one orbit. In these data, we have discovered 37 candidate transient events, of which five are likely cluster $\mathrm{SNe}$ Ia, eight are possible cluster $\mathrm{SNe} \mathrm{Ia}$, and the rest are background or foreground events. We have determined cluster membership of the candidate host galaxies using follow-up spectroscopy from ground-based telescopes, and have measured stellar luminosities using Subaru and HST photometry.

We find that the $\mathrm{SN}$ rate in clusters at $0.5<z<0.9$, which we have measured here accurately for the first time, is consistent with the rates measured at lower redshifts. Our main finding is thus that there is little or no evolution in cluster $\mathrm{SN}$ rates from the present time out to $z \approx 0.9$. The low and unevolving $\mathrm{SN}$ rate suggests that an increase in ICM iron abundance between redshift 0 and 1 , as reported based on X-ray observations, if real, is the result of the redistribution of iron in clusters, and not due to the production of new iron by SNe during this period. Two of the candidate events are possible hostless cluster SNe Ia, which we have discussed in the context of the few known examples of such intergalactic $\mathrm{SNe}$, and their fraction in clusters.

In a forthcoming paper, we will combine our current result with previous measurements to analyze the cluster SN Ia rate as a function of redshift, to examine the clues it can provide regarding the progenitors of $\mathrm{SNe} \mathrm{Ia}$, and to investigate in more detail the role of SNe Ia in the metal enrichment of the ICM.

Finally, although the result presented in this paper is the most accurate cluster rate to date at high redshifts, it still suffers from uncertainties due to the small number of $\mathrm{SNe}$ on which it is based, and the difficulty in acquiring spectroscopic confirmation for $\mathrm{SNe}$ at such redshifts. While spectroscopy will remain challenging in the foreseeable future, upcoming surveys yielding larger numbers of cluster SNe could lessen the current Poisson uncertainties in the rates. This would lead to further progress in the study of several issues that can be illuminated by means of cluster $\mathrm{SN}$ rates.

We thank the anonymous referee for very useful comments that greatly improved the paper. We thank D. Poznanski and M. Sullivan for useful discussions and help with the Keck observations. K.S. acknowledges support from the Kersten Visiting Fellowship Fund, and thanks the Department of Astronomy and Astrophysics at the University of Chicago for their hospitality during the time some of this research was conducted. A.G. and J.P.K. acknowledge support by the grant 07AST-F9 from the Ministry of Science, Culture \& Sport, Israel, \& the Ministry of Research, France. A.G. is also supported by the Israeli Science Foundation, an EU Seventh Framework Programme Marie Curie IRG fellowship, and the Benoziyo Center for Astrophysics, a research grant from the Peter and Patricia Gruber Awards, and the William Z. and Eda Bess Novick New Scientists Fund at the Weizmann Institute. D.M. acknowledges support by the Israel Science Foundation. This research was supported by National Science Foundation grants AST-0607485 and AST-0908886 to A.V.F., as well as by NASA/HST grant GO-10793 from the Space Telescope Science Institute (STScI), which is operated by AURA, Inc., under NASA contract NAS 5-26555. A.V.F. is also grateful for the Department of Energy grant DE-FG02-08ER41563. The authors wish to recognize and acknowledge the very significant cultural role and reverence that the summit of Mauna Kea (where the Subaru and Keck data were obtained) has always had within the indigenous Hawaiian community; we are most fortunate to have the opportunity to conduct observations from this mountain.

\section{REFERENCES}

Allen, C. W. 1973, Astrophysical Quantities (3rd ed.; London: Univ. London, Athlone Press)

Allred, J. C., Hawley, S. L., Abbett, W. P., \& Carlsson, M. 2006, ApJ, 644, 484

Aubourg, É., Tojeiro, R., Jimenez, R., Heavens, A., Strauss, M. A., \& Spergel, D. N. 2008, A\&A, 492, 631

Bai, L., et al. 2007, ApJ, 664, 181

Balestra, I., Tozzi, P., Ettori, S., Rosati, P., Borgani, S., Mainieri, V., Norman, C., \& Viola, M. 2007, A\&A, 462, 429

Barbary, K. H., et al. 2010, BAAS, 41, 449

Bardeau, S., Kneib, J.-P., Czoske, O., Soucail, G., Smail, I., Ebeling, H., \& Smith, G. P. 2005, A\&A, 434, 433

Bell, E. F., \& de Jong, R. S. 2001, ApJ, 550, 212

Bell, E. F., McIntosh, D. H., Katz, N., \& Weinberg, M. D. 2003, ApJS, 149, 289

Bertin, E., \& Arnouts, S. 1996, A\&AS, 117, 393

Borgani, S., Diaferio, A., Dolag, K., \& Schindler, S. 2008a, Space Sci. Rev., 134, 269

Borgani, S., Fabjan, D., Tornatore, L., Schindler, S., Dolag, K., \& Diaferio, A. 2008b, Space Sci. Rev., 134, 379

Borgani, S., et al. 2004, MNRAS, 348, 1078

Botticella, M. T., et al. 2008, A\&A, 479, 49

Brandt, T. D., Tojeiro, R., Aubourg, É., Heavens, A., Jimenez, R., \& Strauss, M. A. 2010, arXiv: 1002.0848

Bruzual, G., \& Charlot, S. 2003, MNRAS, 344, 1000

Butcher, H., \& Oemler, A., Jr. 1978, ApJ, 219, 18

Butcher, H., \& Oemler, A., Jr. 1984, ApJ, 285, 426

Cappellaro, E., Evans, R., \& Turatto, M. 1999, A\&A, 351, 459

Cappellaro, E., et al. 2005, A\&A, 430, 83

Cignoni, M., Ripepi, V., Marconi, M., Alcalà, J. M., Capaccioli, M., Pannella, M., \& Silvotti, R. 2007, A\&A, 463, 975

Clemens, M. S., Alexander, P., \& Green, D. A. 2000, MNRAS, 312, 236

Dahlen, T., Strolger, L.-G., \& Riess, A. G. 2008, ApJ, 681, 462

Dahlen, T., et al. 2004, ApJ, 613, 189

Dawson, K. S., et al. 2009, AJ, 138, 1271

de Plaa, J., Werner, N., Bleeker, J. A. M., Vink, J., Kaastra, J. S., \& Méndez, M. 2007, A\&A, 465, 345

De Young, D. S. 1978, ApJ, 223, 47

Dilday, B., et al. 2010, ApJ, 715, 1021

Donovan, D. A. K. 2007, PhD thesis, Univ. Hawaii

Dubinski, J. 1998, ApJ, 502, 141

Eason, E. L. E., Giampapa, M. S., Radick, R. R., Worden, S. P., \& Hege, E. K. 1992, AJ, 104, 1161

Ebeling, H., Barrett, E., Donovan, D., Ma, C.-J., Edge, A. C., \& van Speybroeck, L. 2007, ApJ, 661, L33

Ebeling, H., Jones, L. R., Fairley, B. W., Perlman, E., Scharf, C., \& Horner, D. 2001, ApJ, 548, L23

Ehlert, S., \& Ulmer, M. P. 2009, A\&A, 503, 35

Faber, S. M., et al. 2003, Proc. SPIE, 4841, 1657

Förster, F., \& Schawinski, K. 2008, MNRAS, 388, L74

Förster, F., Wolf, C., Podsiadlowski, P., \& Han, Z. 2006, MNRAS, 368, 1893

Galama, T. J., et al. 1998, Nature, 395, 670

Gal-Yam, A., \& Maoz, D. 2000a, in Large Scale Structure in the X-ray Universe, ed. M. Plionis \& I. Georgantopoulos (Paris: Atlantisciences), 359

Gal-Yam, A., \& Maoz, D. 2000b, in AIP Conf. Proc. 522, Cosmic Expansions: Tenth Astrophysics Conference, ed. W. W. Zhang \& S. S. Holt (Melville, NY: AIP), 107

Gal-Yam, A., \& Maoz, D. 2004, MNRAS, 347, 942

Gal-Yam, A., Maoz, D., Guhathakurta, P., \& Filippenko, A. V. 2003, AJ, 125 , 1087

Gal-Yam, A., Maoz, D., Guhathakurta, P., \& Filippenko, A. V. 2008, ApJ, 680, 550

Gal-Yam, A., Maoz, D., \& Sharon, K. 2002, MNRAS, 332, 37

Gehrels, N. 1986, ApJ, 303, 336

Giavalisco, M., et al. 2004, ApJ, 600, L93

Goto, T., et al. 2002, PASJ, 54, 515

Gould, A., Flynn, C., \& Bahcall, J. N. 1998, ApJ, 503, 798

Graham, M. L., et al. 2008, AJ, 135, 1343

Gunn, J. E., \& Gott, J. R. I. 1972, ApJ, 176, 1

Guy, J., Astier, P., Nobili, S., Regnault, N., \& Pain, R. 2005, A\&A, 443, 781

Hakobyan, A. A., Petrosian, A. R., McLean, B., Kunth, D., Allen, R. J., Turatto, M., \& Barbon, R. 2008, A\&A, 488, 523

Hamuy, M., Trager, S. C., Pinto, P. A., Phillips, M. M., Schommer, R. A., Ivanov, V., \& Suntzeff, N. B. 2000, AJ, 120, 1479

Hansen, S. M., Sheldon, E. S., Wechsler, R. H., \& Koester, B. P. 2009, ApJ, 699, 1333

Hjorth, J., et al. 2003, Nature, 423, 847 
Howell, D. A. 2001, ApJ, 554, L193

Kartaltepe, J. S., Ebeling, H., Ma, C. J., \& Donovan, D. 2008, MNRAS, 389, 1240

Kay, S. T., da Silva, A. C., Aghanim, N., Blanchard, A., Liddle, A. R., Puget, J.-L., Sadat, R., \& Thomas, P. A. 2007, MNRAS, 377, 317

Kinney, A. L., Calzetti, D., Bohlin, R. C., McQuade, K., Storchi-Bergmann, T., \& Schmitt, H. R. 1996, ApJ, 467, 38

Knop, R. A., et al. 2003, ApJ, 598, 102

Koekemoer, A. M., Fruchter, A. S., Hook, R. N., \& Hack, W. 2002, in The 2002 HST Calibration Workshop (Baltimore, MD: STScI), 337

Koester, B. P., et al. 2007, ApJ, 660, 239

Kowalski, A. F., Hawley, S. L., Hilton, E. J., Becker, A. C., West, A. A., Bochanski, J. J., \& Sesar, B. 2009, AJ, 138, 633

Kuznetsova, N., et al. 2008, ApJ, 673, 981

Law, N. M., et al. 2009, PASP, 121, 1395

Leibundgut, B. 2001, ARA\&A, 39, 67

Lin, Y.-T., \& Mohr, J. J. 2004, ApJ, 617, 879

Loh, Y.-S., Ellingson, E., Yee, H. K. C., Gilbank, D. G., Gladders, M. D., \& Barrientos, L. F. 2008, ApJ, 680, 214

Malesani, D., et al. 2004, ApJ, 609, L5

Mannucci, F., Della Valle, M., Panagia, N., Cappellaro, E., Cresci, G., Maiolino, R., Petrosian, A., \& Turatto, M. 2005, A\&A, 433, 807

Mannucci, F., Della Valle, M., \& Panagia, N. 2006, MNRAS, 370, 773

Mannucci, F., Maoz, D., Sharon, K., Botticella, M. T., Della Valle, M., Gal-Yam, A., \& Panagia, N. 2008, MNRAS, 383, 1121

Maoz, D. 2008, MNRAS, 384, 267

Maoz, D., \& Badenes, C. 2010, arXiv:1003.3031

Maoz, D., \& Gal-Yam, A. 2004, MNRAS, 347, 951

Maoz, D., Mannucci, F., Li, W., Filippenko, A. V., Della Valle, M., \& Panagia, N. 2010a, MNRAS, in press (arXiv:1002.3056)

Maoz, D., Sharon, K., \& Gal-Yam, A. 2010b, ApJ, submitted (arXiv:1006.3576)

Maughan, B. J., Jones, C., Forman, W., \& Van Speybroeck, L. 2008, ApJS, 174, 117

Mazzali, P. A., Röpke, F. K., Benetti, S., \& Hillebrandt, W. 2007, Science, 315 , 825

Melbourne, J., et al. 2007, AJ, 133, 2709

Miller, C. J., et al. 2005, AJ, 130, 968

Miyazaki, S., et al. 2002, PASJ, 54, 833

Mori, M., \& Burkert, A. 2000, ApJ, 538, 559

Nagai, D., Kravtsov, A. V., \& Vikhlinin, A. 2007, ApJ, 668, 1

Neill, J. D., et al. 2006, AJ, 132, 1126
Nugent, P., Kim, A., \& Perlmutter, S. 2002, PASP, 114, 803

Ofek, E. O., Maoz, D., Rix, H.-W., Kochanek, C. S., \& Falco, E. E. 2006, ApJ, 641,70

Oguri, M., et al. 2004, ApJ, 605, 78

Oke, J. B., et al. 1995, PASP, 107, 375

Pian, E., et al. 2006, Nature, 442, 1011

Perlmutter, S., et al. 1999, ApJ, 517, 565

Phillips, M. M. 1993, ApJ, 413, L105

Porter, S. C., Raychaudhury, S., Pimbblet, K. A., \& Drinkwater, M. J. 2008, arXiv:0804.4177

Poznanski, D., et al. 2007, MNRAS, 382, 1169

Pritchet, C. J., Howell, D. A., \& Sullivan, M. 2008, ApJ, 683, L25

Raskin, C., Scannapieco, E., Rhoads, J., \& Della Valle, M. 2009, ApJ, 707, 74

Rau, A., et al. 2009, PASA, 121, 1334

Renzini, A. 1997, ApJ, 488, 35

Renzini, A. 2006, ARA\&A, 44, 141

Saintonge, A., Tran, K.-V. H., \& Holden, B. P. 2008, ApJ, 685, L113

Scannapieco, E., \& Bildsten, L. 2005, ApJ, 629, L85

Scannapieco, C., Tissera, P. B., White, S. D. M., \& Springel, V. 2008, MNRAS, 389, 1137

Schechter, P. 1976, ApJ, 203, 297

Sharon, K., Gal-Yam, A., Maoz, D., Filippenko, A. V., \& Guhathakurta, P. 2007, ApJ, 660, 1165

Sivanandam, S., Zabludoff, A. I., Zaritsky, D., Gonzalez, A. H., \& Kelson, D. D. 2009, ApJ, 691, 1787

Stanek, K. Z., et al. 2003, ApJ, 591, L17

Sullivan, M., et al. 2006a, AJ, 131, 960

Sullivan, M., et al. 2006b, ApJ, 648, 868

Tornatore, L., Borgani, S., Dolag, K., \& Matteucci, F. 2007, MNRAS, 382, 1050

Totani, T., Morokuma, T., Oda, T., Doi, M., \& Yasuda, N. 2008, PASJ, 60, 1327

Tran, K.-V. H., Kelson, D. D., van Dokkum, P., Franx, M., Illingworth, G. D., \& Magee, D. 1999, ApJ, 522, 39

van der Wel, A., Franx, M., van Dokkum, P. G., Rix, H.-W., Illingworth, G. D., \& Rosati, P. 2005, ApJ, 631, 145

van Dokkum, P. G., Franx, M., Fabricant, D., Illingworth, G. D., \& Kelson, D. D. $2000, \mathrm{ApJ}, 541,95$

Visvanathan, N., \& Sandage, A. 1977, ApJ, 216, 214

White, R. E., III. 1991, ApJ, 367, 69

Woosley, S. E., \& Bloom, J. S. 2006, ARA\&A, 44, 507

Zhilyaev, B. E., et al. 2007, A\&A, 465, 235 